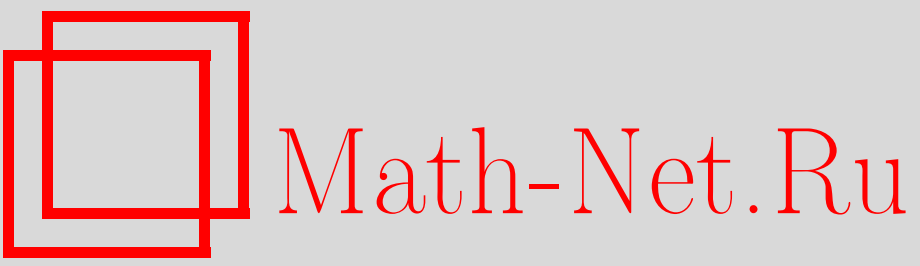

В. В. Белов, С. Ю. Доброхотов, Т. Я. Тудоровский, Асимптотические решения нерелятивистских уравнений квантовой механики в искривленных нанотрубках. I. Редукция к пространственно-одномерным уравнениям, ТМФ, 2004, том 141, номер 2, 267-303

DOI: https://doi.org/10.4213/tmf120

Использование Общероссийского математического портала Math-Net.Ru подразумевает, что вы прочитали и согласны с пользовательским соглашением

http: //www.mathnet.ru/rus/agreement

Параметры загрузки:

IP : 18.209 .158 .208

26 апреля 2023 г., 18:16:11 


\section{АСИМПТОТИЧЕСКИЕ РЕШЕНИЯ НЕРЕЛЯТИВИСТСКИХ УРАВНЕНИЙ КВАНТОВОЙ МЕХАНИКИ В ИСКРИВЛЕННЫХ НАНОТРУБКАХ. I. РЕДУКЦИЯ К ПРОСТРАНСТВЕННО-ОДНОМЕРНЫМ УРАВНЕНИЯМ}

Рассматриваются уравнения нерелятивистской квантовой механики в тонких трехмерных трубках (нанотрубках). Предложен вариант адиабатического приближения, позволяющий редуцировать исходные трехмерные уравнения к одномерным для широкого диапазона энергий продольного движения. Предложенный метод редукции - операторный метод разделения переменных - опирается на операторный метод Маслова. Проведена классификация решений редуцированного одномерного уравнения. Предлагаемая часть I статьи посвящена задаче редукции и описанию основных идей операторного разделения переменных (в адиабатическом приближении) и выводу редуцированных уравнений. Часть II будет посвящена различным асимптотическим решениям и некоторым вытекающим из них эффектам.

Ключевые слова: нанотрубки, адиабатическое приближение, размерное квантование, спин-орбитальное взаимодействие, квазиклассическое приближение.

\section{1. ВВЕДЕНИЕ}

1.1. Нанотрубками называют протяженные молекулы, состояшие из большого числа атомов, расположенных на цилиндрообразных пространственных поверхностях. Поверхность нанотрубок может обладать дополнительным внутренним кручением (рис. 1). Практическая возможность синтезирования таких молекул появилась сравнительно недавно [1]. Первыми были открыты углеродные нанотрубки [2] - разновидности фуллеренов [1]. В настоящий момент синтезированы различные нанотрубки, в том числе легированные металлом [3]. Интерес, который привлекают к себе нанотрубки, связан с их исключительными механическими и электрическими свойствами. В зависимости от диаметра и спиральности нанотрубки могут быть как проводниками, так и полупроводниками.

\footnotetext{
* Московский государственный институт электроники и математики, Москва, Россия. E-mail: belov@miem.edu.ru

${ }^{\dagger}$ Институт проблем механики РАН, Москва, Россия. E-mail:dobr@ipmnet.ru; timtudor@mail.ru
} 

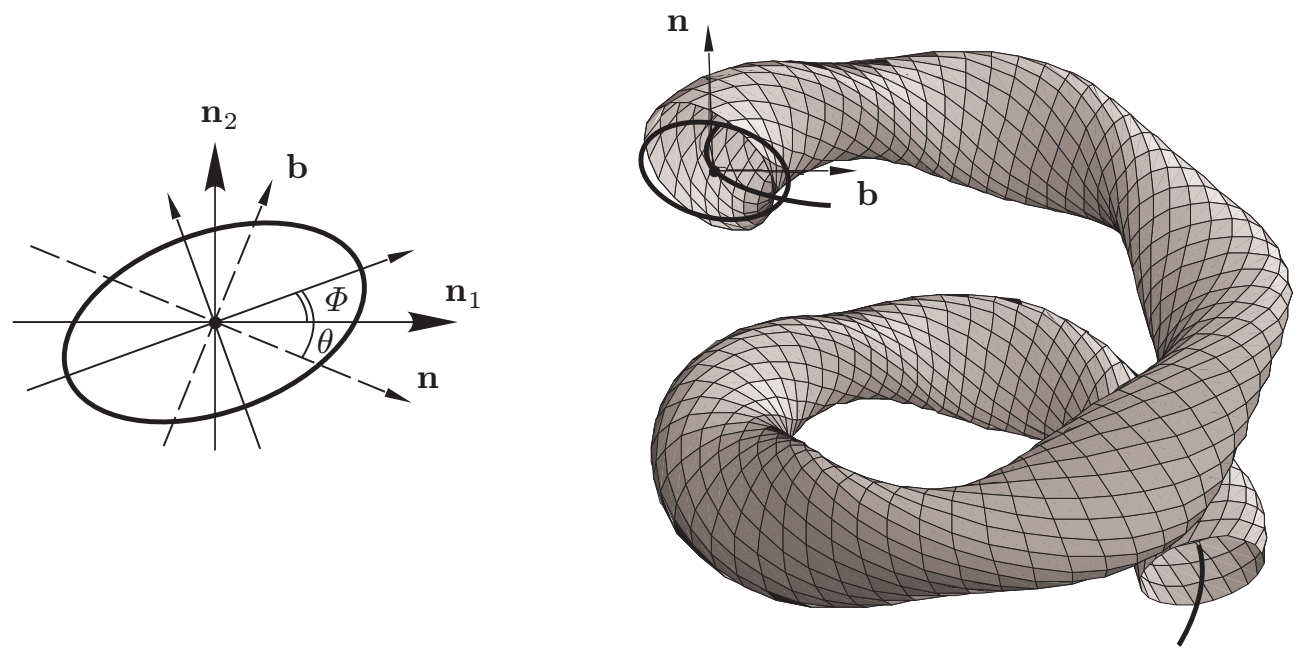

Рис. 1

Слабость электрон-фононного взаимодействия в нанотрубках приводит к упругому баллистическому транспорту электрона на большие расстояния [1], [4]. В связи с этим представляет интерес изучение баллистического транспорта электрона в нанотрубках с учетом их реальной пространственной геометрии и возможных спиновых эффектов.

В приближении метода сильной связи эффективная динамика квантовых состояний определяется нерелятивистским одночастичным гамильтонианом - оператором Шредингера, или, если принимаются во внимание связанные со спином эффекты, оператором Паули с учетом спин-орбитального взаимодействия:

$$
\widehat{\mathcal{H}}=\frac{\widehat{\mathbf{P}}^{2}}{2 m}+v_{\text {int }}(\mathbf{r})+v_{\text {ext }}(\mathbf{r}, t)-\frac{e \hbar}{2 m c}\langle\boldsymbol{\sigma}, \mathbf{H}\rangle+\widehat{\mathcal{H}}_{\mathrm{SO}}, \quad x \in \mathbb{R}^{3},
$$

где $\mathbf{r}$ - радиус-вектор точки, $\hbar$ - постоянная Планка, $\widehat{\mathbf{P}}=-i \hbar \nabla-(e / c) \mathbf{A}(\mathbf{r}, t), \widehat{\mathbf{P}}$ - квазиимпульс, $e=-e_{0}$ - заряд электрона, $m$ - эффективная масса квазичастицы, $v_{\text {int }}(\mathbf{r})$ - потенциал взаимодействия с кристаллом, $v_{\text {ext }}(\mathbf{r}, t), \mathbf{A}(\mathbf{r}, t)$ - потенциалы внешних полей, $\mathbf{H}=\operatorname{rot} \mathbf{A}(\mathbf{r}, t)$ - магнитное поле, $\boldsymbol{\sigma}=\left\{\sigma_{1}, \sigma_{2}, \sigma_{3}\right\}$ - стандартные матрицы Паули, $\widehat{\mathcal{H}}_{\mathrm{SO}}=\alpha\left\langle\boldsymbol{\sigma},\left[\nabla v_{\text {int }}, \widehat{\mathbf{P}}\right]\right\rangle-$ оператор взаимодействия спина электрона с электрическим полем кристалла. Постоянная $\alpha$ зависит от типа рассматриваемого кристалла [5]. Жирными буквами обозначены векторы и векторные операторы с компонентами в декартовой системе координат. В частности, при условии $v_{\text {int }}(x)=0$ гамильтониан (1.1) отвечает модели "пустого квантового цилиндра", помешенного во внешнее поле.

Диаметр нанотрубки $d_{0} \sim 1$ нм $(10 \AA)$ сопоставим с дебройлевской длиной волны электрона $\lambda=2 \pi / k_{\mathrm{F}} \sim 1$ нм с энергией порядка энергии Ферми $\varepsilon_{\mathrm{F}} \sim 1$ эВ, ее характерная длина $l_{0}$ оказьвается сушественно больше $d_{0}$ (например, $l_{0} \sim 100 \mathrm{Hм}$, см. [3]). Это обстоятельство приводит к эффекту “размерного квантования”: область локализации волновой функции по направлениям, нормальным оси трубки, имеет порядок $\lambda$, и энергия, отвечаюшая движению вдоль этих направлений, квантуется. В силу этого факта 
полная трехмерная задача описания квантовых состояний разбивается на ряд редуцированных задач (на “подзонах размерного квантования") с одномерными эффективными квантовыми гамильтонианами (на оси трубки), что, в конечном итоге, позволяет эти состояния описывать достаточно явно с помощью асимптотических формул.

Основная цель этой работы - построить приближенные (асимптотические) решения следуюших задач: для нестационарного уравнения $i \hbar \Psi_{t}=\widehat{\mathcal{H}} \Psi-$ задачи об эволюиии волнового пакета специального вида (начально-краевой задачи в области $\Omega \in \mathbb{R}^{3}$, занимаемой трубкой); для стационарного уравнения $\widehat{\mathcal{H}} \psi=E \psi-$ задачи построения связанных состояний электрона (спектральной задачи, $\psi \in L_{2}(\Omega)$ ) и задачи упругого рассеяния $\left(E=k^{2},|\psi|<\mathrm{const}\right)$.

1.2. Локализация состояния в трубке следует из наличия соответствующих ограничений, например "мягких" или “жестких" стенок (см. [6]-[8], а также п. 2.3). Таким образом, нанотрубки можно рассматривать как квантовые системы с ограничениями. В электродинамике, акустике, физике океана объекты такого типа также хорошо известны - это волноводы. Благодаря наличию "разномасштабности" в рассматриваемой квантовой системе в задаче возникает малый параметр $\mu=d_{0} / l_{0}$. Мы будем предполагать, что геометрические характеристики трубки (кривизна и кручение оси, внутреннее кручение и т.д.) могут сушественно изменяться лишь на масштабах $\sim l_{0}$, что позволяет использовать адиабатическое приближение по этому параметру, который естественно называть "адиабатическим". Квантовые системы с ограничениями широко обсуждались в различных аспектах, в том числе и в последнее время [6], [8]-[10]. По-сушеству, в этих работах использованы различные варианты адиабатического приближения, позволяющие исключить ограничения типа "мягких" или “жестких" стенок. В теории волноводов такое исключение эквивалентно асимптотическому разделению колебаний на продольные и поперечные моды. Для уравнения Гельмгольца оно было проделано более 40 лет назад в работе [11], где, в частности, выписано уравнение для продольной моды и показано, что, подбирая кривизну волновода, можно создать резонаторы с одномодовыми связанными состояниями [12]. В дальнейшем в квантово-механических задачах подобные уравнения были выведены в работах [6], [8].

С математической точки зрения асимптотическое разделение на продольные и поперечные моды связано с тем обстоятельством, что наличие различных характерных масштабов приводит при соответствуюшем обезразмеривании к появлению малого параметра перед производной по “медленной” переменной на оси трубки; при “быстрых" поперечных переменных этот параметр отсутствует. Эта ситуация очень близка к задачам физики молекул: роль быстрых переменных играют координаты легких электронов, а медленных - тяжелых ядер; подзоны размерного квантования называются термами. Класс линейных задач, в которых имеются быстрые и медленные переменные, в математической литературе часто называют уравнениями с операторнозначным символом [13]. $\mathrm{K}$ их числу относятся задачи для уравнений, описывающих квантовую механику молекул, электронные волны в кристаллах (уравнения с быстроменяюшимися коэффициентами), внутренние и поверхностные волны в океане, волны в оболочках, интегральную оптику и многие другие волновые процессы (см., например, [12]-[19]).

Разделяя поперечные и продольные движения, следует иметь в виду, что квантовые 
продольные состояния не являются однородными: вдоль оси волновода соответствующие волновые функции могут достаточно медленно меняться, но могут и быстро осциллировать. Такое поведение волновой функции зависит, например, от того, описывает ли она сильно или слабо возбужденное состояние. С другой стороны, наличие внешних электромагнитных полей и нетривиальной геометрии может вызвать быстрые изменения волновых функций даже у невозбужденных состояний. Методы отыскания волновых функций продольного движения в разных ситуациях оказываются различными. В частности, для случая осциллирующих решений естественно применить квазиклассическое приближение. Отражение этого факта состоит в присутствии в задаче наряду с адиабатическим параметром $\mu$ еще одного параметра, характеризующего частоту осцилляций вдоль оси трубки. Этот “квазиклассический” параметр определяется равенством $h=\lambda_{\|} / l_{0}$, где $\lambda_{\|}$- характерная длина волны продольного движения. Если $h \ll 1$, то волновая функция быстро меняется вдоль оси трубки и работает квазиклассическое приближение; если $h \sim 1$, то волновая функция вдоль оси трубки меняется плавно, и для анализа ее поведения следует использовать другие подходы. Через отношение параметров $\mu$ и $h$ можно оценить отношение продольной и поперечной энергий. Характерную величину поперечной энергии $\varepsilon_{\perp}$ в трубке можно найти из соотношения неопределенностей: поскольку “поперечный” импульс $\sim \hbar / d_{0}$, то $\varepsilon_{\perp} \sim \hbar^{2} /\left(m d_{0}^{2}\right)$. Тогда энергия продольного движения $\varepsilon_{\|}=\left(\mu^{2} / h^{2}\right) \varepsilon_{\perp}$.

Быстроменяюшуюся вдоль продольного направления волновую функцию можно сразу искать в исходной трехмерной задаче, применяя осцилляторное приближение в случае ее быстрого убывания (вдоль продольного направления), - тогда это будет приближение, первоначально предложенное Борном и Оппенгеймером [15], или стандартное квазиклассическое приближение в случае ее быстроосциллирующего поведения [10], [13], [20], - тогда волновая функция полностью определяется (вешественным) каноническим оператором Маслова. Наконец, отдельно можно рассмотреть решения, медленно меняющиеся вдоль продольного направления (см., например, [6], [7]). Заметим, что объекты, определяющие асимптотику полной волновой функции, вообше говоря, могут быть существенно различными в зависимости от соотношений между параметрами $\mu$ и $h$. Другой подход состоит в первоначальной “адиабатической” редукции трехмерной задачи к набору одномерных задач с некоторым квантовым эффективным гамильтонианом продольного движения, равномерно по параметрам охватывающих все перечисленные вьше ситуации, и в последуюшем нахождении асимптотических точных решений (одномерных) редуцированных задач и восстановлении по ним полной волновой функции. По-сушеству, в основе второго подхода лежит подстановка Пайерлса [15], явно или неявно активно используемая в уже перечисленных выше задачах физики твердого тела, электромагнетизма и механики сплошных сред. Мы используем второй подход: сначала (в предлагаемой части I данной статьи), опираясь на операторный метод Маслова [21] и предложенное в работах [19] представление решений (4.1) уравнений с операторнозначными символами, формализуюшее подстановку Пайерлса, выводим редуцированные уравнения на подзоне "размерного квантования" (см. (4.2), (4.4)-(4.6)), описываюшие продольные движения с учетом геометрических характеристик нанотрубки и электромагнитных полей и охватываюшие равномерно по параметру $h$ указанные выше 
случаи. Затем (в части II) мы получим различные асимптотические решения редуцированных и исходного уравнений и обсудим различные описываемые этими решениями эффекты (частично аннонсированные в работах [20]). Основной результат части I заключается в формуле (4.5).

Классический аналог редукции первого шага хорошо известен [22]: исключение “жесткого" потенциала по поперечным направлениям приводит в нулевом приближении к системе с голономными связями, эквивалентной одномерной лагранжевой системе. Однако классическая система, отвечающая редуцированной квантовой, вообше говоря, не совпадает с результатом классической редукции. Более того, возникающие при адиабатической редукции классические системы оказываются различными при разных соотношениях между $\mu$ и $h$; пользуясь терминологией работы [14], можно сказать что они соответствуют различным нестандартным характеристикам квантовой задачи. Например, иногда классическое уравнение движения может включать слагаемые, учитьвающие наличие спина. Физическое объяснение последнего факта состоит в том, что продольные движения определяются уже сравнительно малыми энергиями, вполне сравнимыми с энергией спина.

1.3. Хотя используемый нами подход и лежащие в его основе формулы "операторного разделения переменных" (2.6), (4.1) носят "методический” характер и являются модификацией обшего подхода [21], тем не менее он представляется нам весьма полезным в различных адиабатических задачах, поскольку дает общую регулярную схему аккуратного вывода редуцированных уравнений и позволяет качественно и количественно оценить рамки применимости того или иного приближения. Соображения, приводяшие к формулам (2.6), (4.1), классификацию разного рода приближений, отношения между адиабатическими и квазиклассическими асимптотиками и т.д., естественно, лучше объяснить на простом примере. Эти рассуждения, часть из которых хорошо известна физикам, а часть - математикам, собраны в разделе 2 , в который мы включили также некоторые необходимые минимальные сведения из операторного исчисления некоммутируюших операторов [21] (см. также [19]). Подчеркнем, что используемые факты из работ [21] - не просто рассуждения типа "математического оправдания или обоснования", это - вполне конструктивные и развитые алгоритмы, хорошо приспособленные для изучаемых здесь задач. Более того, с математической точки зрения приводимый в части І вывод основных формул выглядит лишь как “схема" и эвристические соображения; необходимые "обоснования" мы переносим в часть II.

Собственно результаты части I излагаются в разделе 4; там же мы приводим одно из естественных элементарных следствий редуцированных уравнений: возможность моделирования с помощью выбора геометрии нанотрубок, помешенных в однородное электрическое поле, различных одномерных потенциалов (типа барьера, одной или двух ям, или периодических и даже квазипериодических). Третий раздел носит вспомогательньй характер: в нем вводятся безразмерные переменные и параметры задачи, дается описание оператора (1.1) в подходяших переменных. Технические детали вывода формул, приведенных в разделах 2-4, вынесены в приложения. 


\section{2. МОДЕЛЬНЫЙ ПРИМЕР: АДИАБАТИЧЕСКОЕ ПРИБЛИЖЕНИЕ И “ОПЕРАТОРНОЕ” РАЗДЕЛЕНИЕ ПЕРЕМЕННЫХ}

2.1. Поперечные и продольные моды. Адиабатический параметр. Проиллюстрируем основные идеи и соображения на примере двумерного уравнения Шредингера в "узком" планарном волноводе $\Omega=\left\{\left(x, y \in \mathbb{R}^{2}\right): 0 \leqslant x \leqslant l_{0}, 0 \leqslant y \leqslant d_{0}\right\}$ :

$$
i \hbar \frac{\partial \Psi}{\partial t}=-\frac{\hbar^{2}}{2 m}\left(\frac{\partial^{2}}{\partial x^{2}}+\frac{\partial^{2}}{\partial y^{2}}\right) \Psi+v(x, y) \Psi .
$$

Здесь $m$ - масса (квази)частицы. Относительно функции $\Psi$ предположим, что она удовлетворяет условию Дирихле на боковых границах волновода: $\left.\Psi\right|_{y=0}=\left.\Psi\right|_{y=d_{0}}=0$. Условия на торцевых границах волновода в конкретных ситуациях формулируются поразному. Например, это может быть также условие Дирихле $\left.\Psi\right|_{x=0}=\left.\Psi\right|_{x=l_{0}}=0$, условие Борна-Кармана $\Psi\left(x+l_{0}, y, t\right)=\Psi(x, y, t)$ или условия, соответствующие рассеянию плоской волны и т.д.

То, что волновод является "узким", означает, что безразмерный параметр $\mu=d_{0} / l_{0}$ оказьвается малым. В дальнейшем будем называть $\mu$ адиабатическим параметром.

Предположим на некоторое время, что потенциал $v$ зависит лишь от продольной переменной $x$. Тогда, разделяя переменные, мы можем найти специальные частные решения уравнения (2.1) в виде $\Psi=\chi^{\nu}(y) \psi^{\nu}(x, t), \nu=1,2, \ldots$ З Здесь $\chi^{\nu}(y)=\sin \left(\nu \pi y / d_{0}\right)-$ собственные функции ("поперечные моды") оператора "поперечной кинетической энергии" $-\left(\hbar^{2} / 2 m\right) \partial^{2} / \partial y^{2}$ с условием Дирихле $\left.\chi\right|_{y=0}=\left.\chi\right|_{y=d_{0}}=0, \nu$-номер поперечной моды и $\psi^{n}(x, t)$ - решение нестацинарного одномерного уравнения Шредингера с потенциалом

$$
v_{\text {eff }}^{\nu}(x)=v(x)+\nu^{2} \frac{\pi^{2} \hbar^{2}}{2 m d_{0}^{2}} .
$$

Второе слагаемое в последней формуле есть энергия "поперечного" движения; при малых $\nu$ она имеет порядок $\varepsilon_{\perp}=\hbar^{2} /\left(m d_{0}^{2}\right)$. Величину $\varepsilon_{\perp}$ можно получить из принципа неопределенности Гейзенберга. Действительно, размер области локализации волновой функции в поперечном направлении равен $d_{0}$, поэтому в силу принципа неопределенности для характерного поперечного импульса имеем $p_{\perp}=\hbar / d_{0}$, и, следовательно, характерная энергия поперечного движения $\sim p_{\perp}^{2} /(2 m)=\varepsilon_{\perp} / 2$.

Энергия $\varepsilon_{\perp}$ и характерные размеры $l_{0}$ и $d_{0}$ порождают характерное время $T=$ $\omega_{\perp}^{-1} l_{0} / d_{0}=m l_{0} d_{0} / \hbar$, где $\omega_{\perp}=\varepsilon_{\perp} / \hbar-$ частота, соответствующая поперечной энергии $\varepsilon_{\perp}$. В случае, когда скорости продольного и поперечного движений имеют одинаковый порядок, частица проходит расстояние $l_{0}$ за это время. Используя величины $\varepsilon_{\perp}, d_{0}$, $l_{0}, T$, удобно и естественно перейти к учитываюшим разные масштабы безразмерным переменным $v^{\prime}, x^{\prime}, y^{\prime}, t^{\prime}$, полагая $v^{\prime}=v / \varepsilon_{\perp}, x^{\prime}=x / l_{0}, y^{\prime}=y / d, t^{\prime}=t / T$.

2.2. Эффективные гамильтонианы и одномерные редуцированные уравнения в подзоне поперечного квантования. В новых переменных уравнение (2.1) примет вид уравнения с малым параметром $\mu$ (штрихи у переменных для краткости опускаем):

$$
i \mu \frac{\partial \Psi}{\partial t}=-\frac{1}{2}\left(\mu^{2} \frac{\partial^{2}}{\partial x^{2}}+\frac{\partial^{2}}{\partial y^{2}}\right) \Psi+v(x, y) \Psi .
$$


Краевые условия на боковых сторонах волновода превратятся в условия: $\left.\Psi\right|_{y=0}=$ $\left.\Psi\right|_{y=1}=0$. В дальнейшем нам удобно временно “забыть" про краевые условия на концах волновода, считая его бесконечно длинным, и понимать под областью, в которой изучается уравнение $(2.2)$, область $\Omega=\left\{(x, y) \in \mathbb{R}^{2},-\infty<x<\infty, 0 \leqslant y \leqslant 1\right\}$. К краевым условиям на концах волновода $\{x=0, x=1\}$ мы вернемся позднее, а сейчас отметим только, что под введенным ранее параметром $l_{0}$ можно понимать не только длину волновода, но и, например, размер области, где волновая функция отличается от монохроматической волны, или область, вне которой волновая функция становится экспоненциально малой (по адиабатическому параметру $\mu$ ).

В новых переменных оператор кинетической энергии "поперечных колебаний” примет вид - $(1 / 2) \partial^{2} / \partial y^{2}$, его собственные функции $\chi^{\nu}(y)$, удовлетворяюшие условиям Дирихле

$$
\left.\chi^{\nu}\right|_{y=0}=\left.\chi^{\nu}\right|_{y=1}=0
$$

- это функции $\chi^{\nu}=\sin (\nu \pi y)$. Уравнение для функции $\psi^{\nu}$ - одномерное уравнение Шредингера на прямой $x \in \mathbb{R}$ с малым параметром $\mu$ :

$$
i \mu \frac{\partial \psi^{\nu}}{\partial t}=\hat{L}^{\nu} \psi^{\nu}
$$

заданное оператором

$$
\hat{L}^{\nu}=-\frac{\mu^{2}}{2} \frac{\partial^{2}}{\partial x^{2}}+v_{\text {eff }}^{\nu}(x)
$$

с потенциалом $v_{\text {eff }}^{\nu}(x)=v(x)+\nu^{2} \pi^{2}$. Оно определяет "продольные моды" $\psi^{\nu}$ на $\nu$-й подзоне поперечного квантования. Если предположить, что $v(x)=$ const, то для уравнения (2.4) можно построить решения в виде плоской волны: $\psi^{\nu}=e^{-i E_{\nu} t / \mu} e^{i k x}$, где $E_{\nu}(k)=\mu^{2} k^{2} / 2+\nu^{2} \pi^{2}+v$ - полная энергия и $k$ - продольное волновое число. Таким образом, мы имеем две разные длины волны: дискретную "поперечную" $\lambda_{\perp}^{\nu}$ и так называемую "продольную" $\lambda_{\|}=k^{-1}$. Понятно, что безразмерный волновой вектор $k$ характеризует относительную скорость продольного движения; имея в виду последующие асимптотические разложения, нам удобно ввести "квазиклассический" параметр $h$, соответствующий порядку безразмерной переменной $\lambda_{\|}$, т.е. $\lambda_{\|} \sim h$. Параметр $h$, как и параметр $\mu$, будет играть важную роль в дальнейшем; подробно смысл введения этого параметра мы обсудим в п. 2.6 .

Любое решение уравнения (2.4) можно представить в виде интеграла Фурье от произведения экспоненты на функцию, зависяшую от $k$. Суммируя все решения, соответствуюшие разным номерам $\nu$, можно получить широкий класс решений исходного двумерного уравнения Шредингера. С физической точки зрения, однако, решения, соответствующие большим номерам $\nu$, не очень интересны, так как эти энергетические уровни, как правило, пусты. К обсуждению важных следствий из этого факта мы вернемся в конце п. 2.4 .

Предположим теперь, что гладкий потенциал $v$ есть сумма $v_{1}(x)+v_{2}(y)$. Тогда мы можем реализовать предыдушие соображения и редуцировать исходное двумерное уравнение к набору уравнений (2.4), но теперь $\chi^{\nu}$ есть собственная функция оператора $-\partial^{2} / \partial y^{2}+v_{2}(y)$ с условиями (2.3). Отвечаюшие $\chi^{\nu}$ собственные значения обозначим 
$\lambda^{\nu}$. Очевидно, $\chi^{\nu}$ есть также и собственная функция семейства операторов, зависящих от "параметра" $x:-\partial^{2} / \partial y^{2}+v_{1}(x)+v_{2}(y)$. Конечно, введение слагаемого $v_{1}(x)$ в последний оператор возможно из-за того, что оно приводит лишш к сдвигу собственных значений. Используя подобные соображения, мы можем включить в этот оператор слагаемое $p^{2} / 2$, соответствующее производной $-\left(\mu^{2} / 2\right) \partial^{2} / \partial x^{2}$ в уравнении (2.2). Теперь мы можем сказать, что функции $\chi^{\nu}$ есть собственные функции оператора (без "крышки")

$$
\mathcal{H}=\frac{p^{2}}{2}-\frac{\mu^{2}}{2} \frac{\partial^{2}}{\partial y^{2}}+v_{1}(x)+v_{2}(y)
$$

удовлетворяюшие условиям (2.3) и отвечающие собственным значениям

$$
H_{\text {eff }}^{\nu}(p, x)=\frac{p^{2}}{2}+v_{1}(x)+\lambda^{\nu} .
$$

Напомним, что эти собственные значения называются әффективныци гамильтонианами или термами. Очевидно, $H_{\text {eff }}^{\nu}$ есть гамильтониан одномерного движения в эффективном потенциале $v_{\text {eff }}=v_{1}(x)+\lambda^{\nu}$. Тогда $\hat{L}^{\nu}$ в уравнении (2.4) можно интерпретировать как оператор, получаемый из эффективного гамильтониана $H_{\mathrm{eff}}^{\nu}(x, p)$ с помошью подстановки Пайерлса [15] $p \rightarrow \hat{p}=-i \mu \partial / \partial x: \hat{L}^{\nu}=H_{\text {eff }}^{\nu}(x,-i \mu \partial / \partial x)$.

2.3. Жесткие и мягкие стенки. В случае, когда слагаемое $v_{2}(y)$ быстро возрастает вблизи границы, например, если $v_{2}=(y-1 / 2)^{2 m}, m$ - натуральное число, собственные функции оператора $\mathcal{H}$ из $L_{2}\left(R_{x_{2}}\right)$ с малыми номерами $\nu$ быстро убывают при $|y-1 / 2| \rightarrow \infty$ и приближенно удовлетворяют условиям Дирихле с достаточно высокой точностью. (При $m \rightarrow \infty$ рассмотренный потенциал $v_{2}$ переходит в потеншиал, равный нулю на отрезке $0 \leqslant y \leqslant 1$ и бесконечности вне этого отрезка. Такой потенциал моделирует условия (2.3).) Следовательно в этом случае мы можем заменить условие Дирихле условием $\chi^{\nu} \in L_{2}\left(R_{y}\right)$, что эквивалентно условию $\left.\Psi\right|_{x=\text { const }} \in L_{2}\left(R_{y}\right)$. Такой тип условий называется "мягкими стенками" в отличие от условий (2.3), которые назьваются "жесткими стенками". В модельных физических задачах потенциал $v_{2}(y)$ возникает как потенциал конфайнмента в низкоразмерных (кристаллических) системах. Под шириной волновода с мягкими стенками можно понимать, например, величину $d \approx \sqrt{\int_{0}^{1}\left|\chi^{0}(y)\right|^{2}(y-1 / 2)^{2} d y}$ при условии, что $\int_{0}^{1}\left|\chi^{0}(y)\right|^{2} d y=1$.

$\mathrm{C}$ физической точки зрения модели с "мягкими" стенками выглядят более естественно, чем модели с "жесткими” стенками. Однако с математической точки зрения, учитывая убывание собственных функший оператора $\mathcal{H}$, "мягкие" стенки удобно дополнять условиями Дирихле, предполагая, что область $\Omega$, ограниченная "жесткими” стенками, не сильно отличается от "воображаемой" физической границы волновода. В случае "искривленных" волноводов введение "жестких" стенок позволяет не заботиться об однозначности определения потенциала во всем пространстве, корректности введения координат в окрестности оси волновода и т.д.

2.4. Операторная формула адиабатического разделения переменных и подстановка Пайерлса. Символы дифференциальных операторов с параметром. Операторнозначные символы. Если потенциал $v$ не может быть представлен в виде суммы $v_{1}(x)$ и $v_{2}(y)$, то описанная процедура может быть реализована 
только приближенно в предположении малости толшины $d_{0}$ по сравнению с $l_{0}$. Тогда мы имеем адиабатическое приближение, и именно этот подход мы будем использовать в настоящей работе. Обсудим естественное обобшение формулы (2.4). Если $v=v(x, y)$, то собственные функции оператора $\mathcal{H}$ могут зависеть также от переменной $x$. Такая зависимость может быть интерпретирована как зависимость "ширины" волновода от переменной $x$. Аналогичная зависимость эффективного потенциала возникает и в случае переменной толшины пустого волновода с жесткими стенками. В этом случае границы $y=0$ и $y=1$ следует заменить на границы $y=d_{1}(x), y=d_{2}(x)$, где $d_{2}(x)>d_{1}(x)$ - гладкие функции. Тогда в случае "пустого волновода" (т.е. когда $\left.v_{2}=0\right) \lambda^{\nu}(x)=$ $\nu^{2} \pi^{2} /\left(d_{2}(x)-d_{1}(x)\right)^{2}$. Ясно, что учет $\lambda^{\nu}=$ const в формуле (2.5) означает лишш сдвиг продольной энергии, в то время как наличие переменной $\lambda^{\nu}(x)$ влияет на классическую и квантовую динамику, задаваемую этим эффективным гамильтонианом. С другой стороны, в гамильтоновой механике координаты и импульсы равноправны, и, следовательно, может случиться, что эти функции также зависят от импульса $p$ (как и эффективный гамильтониан). Но в квантовой механике импульсу соответствует оператор $\hat{p}=-i \mu \partial / \partial x$, следовательно, в формуле $\Psi=\chi^{\nu}(y) \psi^{\nu}(x, t)$ вместо функции $\chi^{\nu}$ мы должны написать оператор $\hat{\chi}^{\nu}=\chi^{\nu}(\hat{p}, x, y, \mu)$, и окончательно имеем

$$
\Psi(x, y, t, \mu)=\chi^{\nu}(\hat{p}, x, y, t, \mu) \psi^{\nu}(x, t), \quad \nu=1,2, \ldots
$$

Обсудим восстановление оператора $\hat{\chi}^{\nu}$ по функции $\chi^{\nu}$. Процедура такого типа "квантование" - широко обсуждалась как в математической, так и в физической литературе. Для полноты изложения мы напомним здесь сложившуюся математическую терминологию, ограничившись минимальными сведениями, достаточными для целей этой работы. Пусть сначала $R(x, p)=\sum_{l=0}^{k} R_{l}(x) p^{l}$-полином по переменным $p$ с гладкими по $x$ коэффициентами. Эта функция порождает оператор $\widehat{R}=\sum_{k=0}^{l} R_{k}(x)(-i \mu \partial / \partial x)^{k}$. Функция $R(x, p)$ называется символом дифференциального оператора $\widehat{R}$ с параметром $\mu$. Способ построения оператора $\widehat{R}$ по символу $R$, разумеется, не единственный; например, можно построить оператор $\widehat{R}^{\prime}=\sum_{l=1}^{k}(-i \mu \partial / \partial x)^{l} R_{l}(x)$, отличный от $\widehat{R}$, поскольку операторы $\hat{p}=-i \mu \partial / \partial x$ и $x$ не коммутируют. Пользуясь обозначениями Фейнмана, можно написать $\widehat{R}=R(\hat{p}, \stackrel{2}{x})$ и $\widehat{R}=R(\stackrel{2}{\hat{p}, \stackrel{1}{x})}$, где цифры над $\hat{p}$ и $x$ определяют порядок их действия (о других способах упорядочивания, например по Вейлю, см. [21] и другие работы). В настоящей работе мы будем всегда использовать первый способ упорядочивания, не оговаривая это в дальнейшем. При таком соглашении задание дифференциального оператора эквивалентно заданию его символа. Устремляя порядок полинома $k$ к бесконечности, можно определить, по крайней мере наивно, операторы, символы которых не являются полиномами. Такие операторы назьваются псевдодифференциальными. Их строгое определение можно дать с помошью преобразования Фурье (зависяшего от параметра $\mu$, см. приложение 2). Соответствуюшие формулы в многомерном случае, формулы для операторов, заданных на многообразии, условия, позволяющие построить по символу оператор, и т.д. можно найти, например, в работах [21]. Замена операторов на их символы оказывается чрезвычайно полезной в практических вычислениях: в результате вычисления, связанные с операторами, заменяются на существенно более прос- 
тую и алгоритмизуемую работу с символами, т.е. функциями ("с $c$-числами"). Поскольку в асимптотических подходах задание оператора практически эквивалентно заданию его символа, то в процессе получения асимптотических формул можнн ограничиться лищь манипуляциями с символами и “вспомнить" об операторах, отвечающих этим символам, при рассмотрении тонких вопросов, например, связанньх с обоснованием асимптотической точности построенных решений. Конечно, основные сложности в работе с функциями от операторов порождены тем фактом, что операторы $\hat{p}$ и $x$ не коммутируют. С другой стороны, их коммутатор равен $i \mu$ и оказывается малым, что позволяет применить в конструкциях асимптотические разложения. В силу этого же факта естественно рассматривать символы $R$, зависяшие от параметров $\mu$, и считать, что $R(p, x, \mu)=R_{0}(p, x)+\mu R_{1}(p, x)+\cdots$, причем понимать правую часть в этом равенстве как асимптотическое разложение по параметру $\mu$. Функцию $R_{0}(p, x)$ называют старшим символом или иногда гамильтонианом, $R_{j}-$ поправками $j$-порядка.

Следуюшее обобшение заключается в предположении, что символ $R$ может зависеть и от других параметров и переменных. Именно такая ситуация возникает в представлении решения $\Psi$ с помошью формулы (2.6) символ оператора $\chi^{\nu}$ наряду с переменными $(p, x)$ зависит и от поперечной переменной $y$ (и параметра $\mu$ ). Следуя сказанному, мы будем считать, что его символ представляется в виде асимптотического разложения

$$
\chi^{\nu}=\chi_{0}^{\nu}+\mu \chi_{1}^{\nu}+\mu^{2} \chi_{2}^{\nu}+\cdots
$$

Мы пока никак не зафиксировали выбор функции $\psi^{\nu}(x, t)$ в формуле (2.6). Обобшая рассуж дения, приведенные в п. 2.2, потребуем, чтобы эта функция удовлетворяла уравнению (2.4), но уже с оператором $L^{\nu}$, символ которого также представляется в виде асимптотического ряда

$$
L^{\nu}=L_{0}^{\nu}(p, x)+\mu L_{1}^{\nu}(p, x)+\mu^{2} L_{2}^{\nu}(p, x)+\cdots, \quad L_{0}^{\nu}(p, x)=H_{\mathrm{eff}}^{\nu}
$$

Старшая часть $L^{\nu}$ - функция $L_{0}^{\nu}(p, x)$ - и есть эффективньй гамильтониан на $\nu$-й подзоне размерного квантования. Естественно, он оказывается равным гамильтониану $H_{\text {eff }}^{\nu}$, определенному формулой (2.5). Оператор $\hat{\chi}^{\nu}$ - это так называемый “сплетающий оператор" на подзоне размерного квантования, задаваемой “термом" (гамильтонианом) $L_{0}^{\nu}$ (ср. с [21]). В смысле сказанного представление (2.6) вместе с уравнением (2.4) и есть формализация адиабатического разделения переменных и подстановки Пайерлса. Разумеется, поправки $L_{1}^{\nu}, L_{2}^{\nu}$ возникают в задачах, где переменные точно не разделяются.

Наличие малого параметра $\mu$ в наших конструкциях и определениях принципиально важно. Например, если с этой точки зрения определить символ оператора в правой части (2.2), то он оказывается равным опять оператору $\mathcal{H}$ (по переменной $y$ ), поскольку перед производными по $x$ содержится малый параметр, а перед производными по $y$ - нет. Уравнения такого типа в математической литературе называют уравнениями $c$ операторнозначным символом. Переменные первого типа (т.е. $x$ в рассматриваемом примере) называют медленными, а второго ( $y$ в нашем примере) - быстрыми. Другие примеры уравнений с операторнозначными символами дают векторные уравнения: в 
этих случаях символ исходного оператора есть матрица. Разумеется, возможны и более сложные ситуации, когда символ исходного оператора есть матрица, коэффициенты которой - дифференциальные операторы по быстрым переменным $y$. K такому классу относятся рассматриваемые в этой работе задачи, порожденные оператором (1.1).

Физический смысл зависимости символа $\chi^{\nu}(p, x, y)$ от переменных $x$ и $p$ заключается в том, что “быстрая" (поперечная) мода адиабатически подстраивается под “медленную" продольную. Конечно, иногда функция $\chi^{\nu}$ или несколько первых членов $\chi_{0}^{\nu}, \ldots, \chi_{k}^{\nu}$ ее разложения в ряд могут не зависеть от $p$ или $x$. Например, если задача достаточно близка (по параметру $\mu$ ) к задаче с разделяюшимися переменными, т.е. к задаче, где "поперечная" и "продольная" моды независимы в первом приближении, то по крайней мере нулевой член разложения $\chi_{0}^{\nu}$ не будет зависеть от $p$. Однако появление зависимости от $p$ в последуюших членах разложения $\chi^{\nu}$ по параметру $\mu$ оказывается весьма сушественным не только для правильного построения оператора $\hat{L}^{\nu}$ редуцированного уравнения (2.4), но и для оценки области применимости адиабатического приближения и количества заполненных энергетических уровней.

Таким образом, мы видим, что решение исходного уравнения может быть разделено на две части: 1) “операторное (адиабатическое) разделение переменных", основанное на формуле (2.6), которое редуцирует исходное уравнение к уравнению (2.4), и 2) решение этого более простого уравнения.

Мы уже говорили, что с физической точки зрения представляют интерес редуцированные уравнения только с малыми номерами $\nu$, и обычно достаточно сосредоточить внимание на случае нескольких $\nu$ (в пространственных аналогах волноводов - нанотрубках - $\nu$, как правило, не превосходит 7 [1]). Этот факт оказьвается очень важным в дальнейшем при рассмотрении уравнений в изогнутых волноводах и трубках и позволяет не заниматься вопросом о применимости получаемых асимптотических формул при больших $\nu$, а также вопросом о сходимости соответствуюших рядов по номеру $\nu$. По этой причине дополнительные краевые или начальные условия достаточно формулировать уже не для исходного уравнения, а для конечных одномерных (упрощенных) уравнений типа (2.2). Используя физическую терминологию, можно сказать, что представляет интерес изучение продольной динамики для небольшого набора подзон поперечного квантования. Аккуратно соответствуюшие дополнительные условия мы сформулируем для случая пространственных волноводов в части II этой работы (см. [20]), посвященной собственно задаче редукиии, которая состоит в вычислении коэффициентов и анализе $\chi_{0}^{\nu}, \chi_{1}^{\nu}, \ldots, L_{0}^{\nu}, L_{1}^{\nu}, \ldots$ и т.д.

2.5. Внутренние и внешние параметры. В рассматриваемом примере мы неявно предполагаем, что безразмерный потенциал $v$ не зависит от $\mu$. На самом деле в реальных ситуциях бывает естественно считать, что $v$ может зависеть как от $\mu$, так и от других параметров. Эти параметры характеризуют напряженности внешних полей, силу различных взаимодействий и т.д. При этом функции $\chi_{0}^{\nu}, \chi_{1}^{\nu}, \ldots, L_{0}^{\nu}, L_{1}^{\nu}, \ldots$ также будут зависеть от этих параметров. Тем не менее при соответствующих ограничениях формула разделения переменных (2.6) остается справедливой. Для математической строгости указанные параметры задачи следует связать некоторыми соотношениями, привязав их, например, к параметру $\mu$. При этом, однако, следует помнить, что в кон- 
кретных ситуациях все эти параметры - числа, и такие связи могут носить весьма условный характер. По этой причине и во избежание загромождения обозначений явную зависимость от таких параметров мы будем указывать лишш при необходимости.

2.6. Квазиклассический параметр $h$. Число членов в разложении сплетающего оператора $\hat{\chi}$ и оператора $\hat{L}$ можно взять сколь угодно большим. Однако явное вычисление членов этих рядов даже с малыми номерами, как правило, представляет собой сложную задачу. Поэтому естественно ограничиться таким числом слагаемых, которое позволяет правильно определить главный член асимптотики волновой функции или значения энергии.

Понятие “главного" члена асимптотики, однако, может определяться не только адиабатическим параметром $\mu$, но и "квазиклассическим параметром" $h$, который связан с видом эффективного потенциала и с решением редуцированного уравнения (2.4). Поясним это более подробно, используя сначала физические соображения. Для большей наглядности вернемся на некоторое время к размерным переменным.

Наша цель состоит в построении асимптотических решений редуцированного уравнения, описывающих движение вдоль оси трубки в достаточно широком диапазоне продольных энергий. С продольной энергией связана характерная дебройлевская длина волны $\lambda_{\|}=\hbar / p_{\|}$, где $p_{\|}$- размерный импульс продольного движения, и “квазиклассический” параметр $h=\lambda_{\|} / l_{0}$.

Рассмотрим опять пример пустого волновода. Тогда энергия, отвечаюшая $\nu$-му поперечному состоянию, имеет вид $p_{\|}^{2} / 2 m+v_{\text {eff }}(x), v_{\text {eff }}(x)=v_{1}(x)+\nu^{2} \pi^{2} \hbar^{2} /\left(2 m d^{2}\right)$. Учитывая связь между дебройлевской длиной волны и соответствующим импульсом, получаем следующие оценки:

$$
\frac{p_{\|}}{p_{\perp}} \sim \frac{\hbar / \lambda_{\|}}{\hbar / \lambda_{\perp}} \sim \frac{d / l_{0}}{\lambda_{\|} / l_{0}} \sim \frac{\mu}{h} .
$$

Поэтому кинетические энергии продольного и поперечного движения соотносятся как $T_{\|} / T_{\perp} \sim \mu^{2} / h^{2}, T_{\perp} \sim \varepsilon_{\perp}$. Теперь вернемся к безразмерным переменным. Тогда безразмерная продольная кинетическая энергия $T_{\|}=p^{2} / 2 \sim \mu^{2} / h^{2}$. Ясно, что при движении частицы вдоль волновода кинетическая энергия может меняться в результате действия силы $f=-\partial v_{\text {eff }} / \partial x$. Чтобы сила $f$ не разогнала частицу так, что ее кинетическая энергия будет иметь порядок, отличный от $\mu^{2} / h^{2}$, необходимо, чтобы работа этой силы не превосходила по порядку характерной величины кинетической энергии, отвечающей начальному импульсу. В безразмерных переменных расстоянию $\sim l_{0}$ отвечает интервал $\sim 1$, поэтому работа силы $f$ имеет порядок производной $\partial v_{\text {eff }} / \partial x$. Отсюда можно заключить, что эффективный потенциал должен иметь вид $v_{\text {eff }}=v_{\text {eff }}^{0}+$ $\left(\mu^{2} / h^{2}\right) v_{\text {eff }}^{1}(x)$, где $v_{\text {eff }}^{0}=$ const, a $v_{\text {eff }}^{1}(x)$ может, вообще говоря, зависеть регулярным образом от параметров $\mu$ и $h$. Более того, $f$ может быть даже равной нулю, поскольку величина характерного продольного импульса определяется не только переменной частью $v_{\text {eff }}^{1}(x)$ эффективного потенциала, но и “входным" импульсом изучаемого волнового пакета (т.е. градиентом фазы волновой функции в начальный момент времени в задаче 
Коши или импульсом падающей волны в задаче рассеяния). В последнем случае асимптотика волновой функции может быть получена с помощью хорошо известного “борновского" приближения.

ЗАмечАниЕ 1 . Подчеркнем, что $v_{\text {eff }}$ задается как внешним полем, так и полем кристалла. Поэтому указанные выше ограничения приводят, в частности, к предположению о достаточно “медленном" изменении геометрических параметров волновода - кривизны (и кручения) его оси, ширины и т.д.

2.7. Характерный временной масштаб и согласованное с ним редуцированное уравнение. Введенное в п. 2.1 для уравнения (2.2) безразмерное время $t^{\prime}=$ $t / T$ фактически было подстроено под случай $p_{\perp} \sim p_{\|}$, т.е. под случай $\mu / h \sim 1$. Если последнее соотношение не выполнено, то время прохождения частицей волновода, под которым естественно понимать характерное время задачи, определяется как $t_{l_{0}}=(h / \mu) T$. Поэтому вместо $t^{\prime}$ удобно ввести новое безразмерное время $t^{\prime \prime}$ с помошью равенства $t=t_{l_{0}} t^{\prime \prime}$, эквивалентного замене $t^{\prime}=(h / \mu) t^{\prime \prime}$.

Такое переопределение временно́го масштаба согласовано с предыдушими физическими рассуждениями следуюшими преобразованиями в уравнении (2.2). Слагаемое $v_{\text {eff }}^{0}$ приводит лиш к сдвигу (перенормировке) энергии в стационарной задаче, порожденной редуцированным уравнением, или появлению множителя $\exp \left(-i v_{\text {eff }}^{0} t^{\prime} / \mu\right)$ в волновой функции $\psi^{\nu}$ нестационарного уравнения Шредингера (2.2). С учетом сказанного представим решение этого уравнения в виде $\psi^{\nu}=\exp \left(-i v_{\mathrm{eff}}^{0} t^{\prime} / \mu\right) \psi^{\prime \nu}$, где $\psi^{\prime \nu}(x, t)$ - новая неизвестная функция. Поскольку мы считаем, что $p \sim \mu / h$, то естественно поделить уравнение на параметр $\mu^{2} / h^{2}$, что дает в левой части производную $i\left(h^{2} / \mu\right) \partial \psi^{\prime \nu} / \partial t^{\prime}$, которая после указанной замены по времени переходит в $i h \partial \psi^{\prime} \nu / \partial t^{\prime \prime}$. Важно подчеркнуть, что это преобразование касается только времени: переменные $x$ и $y$ не преобразуются. В результате вместо уравнения (2.4) с учетом поправок $L_{1}^{\nu}, L_{2}^{\nu}$ и т.д. мы получим уравнение (штрихи опускаем)

$$
\begin{aligned}
i h \frac{\partial \psi^{\nu}}{\partial t}= & \left\{\frac{1}{2}\left(-i h \frac{\partial}{\partial x}\right)^{2}+v_{\mathrm{eff}}^{1}+\right. \\
& \left.+\frac{h^{2}}{\mu}\left[L_{1}^{\nu}\left(\frac{\mu}{h}\left(-i h \frac{\partial}{\partial x}\right), \stackrel{2}{x}\right)+\mu L_{2}^{\nu}\left(\frac{\mu}{h}\left(-i h \frac{\partial}{\partial x}\right), \stackrel{2}{x}\right)+O\left(\mu^{2}\right)\right]\right\} \psi^{\nu} .
\end{aligned}
$$

2.8. О точности асимптотических разложений. Теперь вернемся к вопросу выбора числа членов разложения символов оператора $\hat{L}$ и сплетающего оператора $\hat{\chi}$. Имея в виду наличие в задаче двух параметров $\mu$ и $h$, мы будем вычислять столько членов, сколько нужно для построения главного члена асимптотики по $\max (h, \mu)$, если $h \ll 1$, и по $\mu$, если $h=1$ (напомним, что параметр $\mu$ всегда предполагается малым, а параметр $h$ может быть как малым, так и иметь порядок $O(1))$. Для определения минимально разумного числа членов в асимптотических разложениях естественно опираться на хорошо известную оценку для решения задачи Коши для неоднородного уравнения типа уравнения Шредингера: $i \varepsilon \partial \phi / \partial t=\hat{A} \phi+f,\left.\phi\right|_{t=0}=0$. Здесь $\hat{A}(t)-$ в сушественном самосопряженный при каждом $t$ оператор в $L_{2}, \varepsilon>0$. Пусть $\phi-$ решение этой 
задачи, тогда для любого $t$ из фиксированного отрезка $[0, T]$ справедливо неравенство $\|\phi\|_{L_{2}} \leqslant(T / \varepsilon) \max _{t \in[0, T]}\|f\|_{L_{2}}$.

Предположим, что $\psi_{\text {ex }}$ - точное решение исходного уравнения $(2.2)$ и $\psi_{\text {as }}-$ его асимптотическое решение вида (2.6), совпадаюшие в нулевой момент времени; причем $\psi_{\text {as }}$ удовлетворяет исходному уравнению с невязкой $f_{\text {as }}$. Выберем в качестве оператора $\hat{A}$ исходный квантовый гамильтониан и положим $\varepsilon=\mu$ и $T=(h / \mu) T_{0}$, где $T_{0}$ не зависит от $h$ и $\mu$. Тогда для разности $\phi=\psi_{\mathrm{as}}-\psi_{\mathrm{ex}}$ получим оценку

$$
\left\|\psi_{\text {as }}-\psi_{\text {ex }}\right\| \leqslant \frac{h}{\mu^{2}} T \max \left\|f_{\text {as }}\right\|_{L_{2}} .
$$

Отсюда следует, что минимально разумное число членов в разложении символов операторов $\hat{\chi}$ и $\hat{L}$ в формулах (2.7), (2.8) должно быть по крайней мере таким, чтобы $\left(h / \mu^{2}\right) \times$ $\|f\|_{L_{2}} \ll 1$ при $\mu \ll 1$. Разумеется, следует иметь в виду, что норма невязки $f_{\text {as }}$ зависит от $\mu$ и $h$. Как наводящие соображения мы будем применять оценку (2.10) и к редуцированному уравнению (2.4).

2.9. Классификация квантовых состояний для продольного движения. Вернемся к переходу от уравнения (2.4) к уравнению (2.9). При $h \ll 1$ для построения волновой функции естественно использовать квазиклассическое приближение. Вне окрестности фокальных точек (точек поворота) типичная асимптотика волновой функции с характерной длиной волны $\lambda_{\|} \sim h$ представляется ВКБ-решением

$$
\psi(x, t)=A(x, t, h) \exp \left(\frac{i S(x, t)}{h}\right), \quad A(x, t, h)=A(x, t, 0)+O(h),
$$

где $S(x, t)$ - фаза и $A(x, t, h)$ - вообше говоря, векторная амплитуда. Как известно [13], в первом приближении после подстановки этой функции в исходное уравнение оператор $-i h \partial / \partial x$ в главном заменяется на $\partial S / \partial x$, тем самым порядок слагаемых операторов $h^{2} \mu^{j-2} L_{j}^{\nu}(\stackrel{2}{x},(\mu / h)(-i h \partial / \partial x))$ в уравнении $(2.9)$ определяется порядком функций $L_{j}^{\nu}(x,(\mu / h) \partial S / \partial x)$. Этот факт приводит к также хорошо известному выводу, что фаза $S(x, t)$ определяется классической гамильтоновой системой, гамильтониан которой старшая часть разложения символа по параметру $h$. Имея это в виду, под оператором импульса естественно понимать оператор $\hat{p}^{h}=-i h \partial / \partial x$, а не оператор $\hat{p}=-i \mu \partial / \partial x$. Понятно, что при $\mu=h$ классический гамильтониан - это эффективный гамильтониан (2.5), однако если адиабатический и квазиклассический параметры $\mu$ и $h$ имеют разный порядок, то из представления (2.9) видно, что символ оператора $\hat{L}$ в редуцированном уравнении может быть дополнительно разложен по $\mu / h$. Это мы проделаем позже. Как мы увидим ниже, в некоторых случаях в классический гамильтониан (подчиненный разложению по $h$ ) будут влючаться добавки из $L_{1}^{\nu}$.

Обсудим теперь вопрос о числе членов в разложении оператора в правой части уравнения (2.9), нужного для нахождения старшей части его асимптотического решения. Полагая $\varepsilon=h$ и применяя оценку (2.10) к уравнению (2.9), мы видим, по крайней мере на интуитивном уровне, что для этого достаточно вычислить эффективный гамильтониан $\left(p^{h}\right)^{2} / 2+v_{\text {eff }}^{1}$ и первую поправку $L_{1}^{\nu}\left(x,(\mu / h) p^{h}\right)$. Этот вывод согласуется с хорошо 
известным фактом из теории квазиклассических асимптотик: слагаемые порядка $h^{2}$ (и даже $\left.h^{1+\delta}, \delta>0\right)$ не влияют на фазу $S(x, t)$ и старшую часть амплитуды $A(x, t, 0)$. Этот факт носит общий характер и оказывается всегда справедливым, если $\mu \leqslant h \ll 1$, т.е. когда работает квазиклассическое приближение. В то же время, как мы только что отмечали, конкретные формулы могут сушественно различаться для ситуаций, когда $\mu$ и $h$ имеют одинаковый порядок $(\mu \sim h)$ и когда $\mu \ll h$. Если параметр $h \sim 1$, то квазиклассическое приближение не работает, но уравнение (2.9) по-прежнему оказывается справедливым и даже может быть упрощено, хотя в этом случае следует учесть некоторую часть из $L_{2}^{\nu}$. Наличие отмеченных различий приводит к следуюшей классификации асимптотических решений в зависимости от соотношения между параметрами $\mu$ и $h$ (или, что эквивалентно, в зависимости от соотношения между значениями продольной $T_{\|}$и поперечной $T_{\perp}$ кинетических энергий в волноводе, см. рис. 2).

1. В случае $h=\mu$ мы имеем стандартную "квазиклассическую" ситуацию [13] или “коротковолновый” режим, когда “продольная" энергия имеет порядок энергии поперечного движения и $d \sim \lambda_{\|} \ll l_{0}$ в размерных переменных. Тогда эффективные адиабатический и квазиклассический гамильтонианы и поправки к ним совпадают, и для определения главного члена асимптотики волновой функции требуется полное описание эффективного гамильтониана и первой поправки.

2. В этом случае, который естественно назвать “средневолновым" режсимом, $\mu \ll$ $h \ll 1$, "продольная" энергия моды сушественно менше "поперечной" и $d<\lambda_{\|} \leqslant \sqrt{l_{0} d}$ в размерных переменных. Тогда, разлагая поправку $L_{1}^{\nu}$ по параметру $\mu / h$, получим для символа оператора

$$
L^{\nu}=\left(\frac{\left(p^{h}\right)^{2}}{2}+v_{\mathrm{eff}}^{1}+\frac{h^{2}}{\mu} L_{1}^{\nu}(x, 0)\right)+h \frac{\partial L_{1}^{\nu}}{\partial p}(x, 0) p^{h}+h \cdot O\left(\frac{\mu}{h}\right) .
$$

Отсюда ясно, что недифференциальная часть $\left(h^{2} / \mu\right) L_{1}^{\nu}(x, 0)$ первой адиабатической поправки в разложении оператора $\hat{L}_{1}^{\nu}$ может переместиться в эффективный квазиклассический гамильтониан. Это хорошо видно, если рассмотреть случай $h=\sqrt{\mu}$, когда эффективный квазиклассический гамильтониан становится равным $\left(p^{h}\right)^{2} / 2+v_{\text {eff }}^{1}+$ $L_{1}^{\nu}(x, 0)$. Более того, при $h^{2} \gg \mu$ слагаемое $\left(h^{2} / \mu\right) L_{1}^{\nu}(x, 0)$ может играть определяющую роль. Тогда рассуждения, аналогичные приведенным в п. 2.6, показывают, что оно "разгонит" частицу в продольном направлении, так что характерный продольный импульс в безразмерных переменных приобретет значение $\sqrt{\mu}$. Иначе говоря, в этом случае в качестве $h$ следует использовать параметр $\sqrt{\mu}$, и мы возврашаемся к только

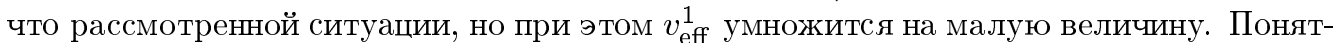
но, что если $L_{1}^{\nu}(x, 0)=0$, то приведенные рассуждения не имеют смысла. Но, как мы увидим ниже, такое слагаемое возникает в нанотрубках как за счет их геометрии, так и за счет внешнего электромагнитного поля. При этом появляются дополнителные параметры - амплитуды полей, которые могут эффективно занизить величину $L_{1}^{\nu}(x, 0)$ и в результате скомпенсировать рост, вызванный параметром $h^{2} / \mu$. Отметим также, что слагаемые в операторе $\hat{L}_{1}^{\nu}$, содержашие вторые и более высокие производные (соответствуюшие высоким степеням разложения $L_{1}^{\nu}$ по переменной $p^{h}$ ), могут быть опушены при вычислении главного члена квазиклассического асимптотического решения, хотя, 


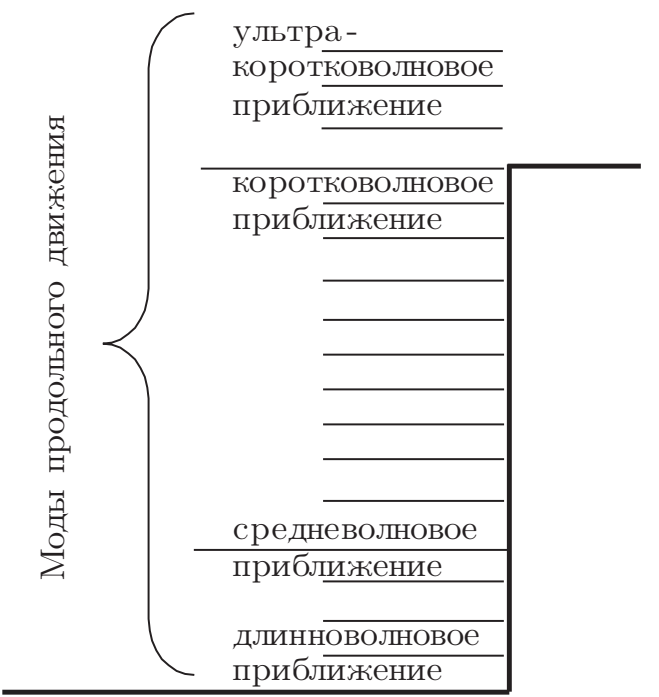

a

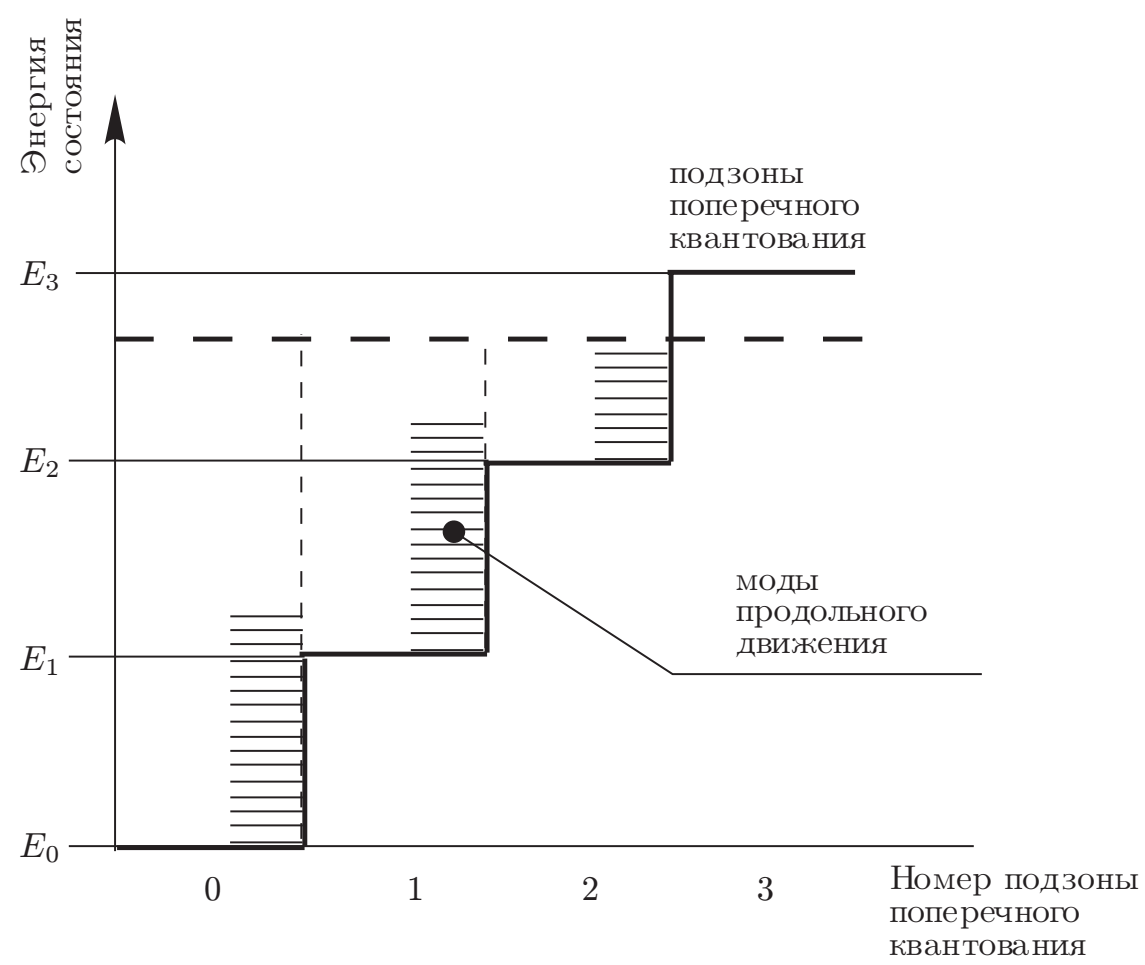

б

Рис. 2 
конечно, их сохранение не ухудшит точность. Вопрос о том, куда включать слагаемое $\left(h^{2} / \mu\right) L_{1}^{\nu}(x, 0)$ при изменении параметра $h$ в пределах $\mu \ll h \ll \sqrt{\mu}$, мы обсудим в п. 2.13 и, в основном, в части II этой статьи.

3. Если параметр $h \sim 1$, то квазиклассическое приближение не применимо, волновые функции если и осциллируют, то довольно медленно, и в размерных переменных $\lambda_{\|} \sim l_{0}$. Такая ситуация возможна, согласно предыдушему, только если $L_{1}^{\nu}(x, 0) \equiv 0$. Но адиабатическое приближение работает, и из (2.9) легко вывести уравнение, описываюшее главный член асимптотики (гладкой) волновой функции $\psi(x, t)$ на оси волновода. Для этого достаточно положить в $(2.9) \quad h=1$ и затем устремить $\mu$ к нулю. В результате такого предельного перехода, который естественно назвать длинноволновы.м приближением, мы приходим к уравнению

$$
i \frac{\partial \psi}{\partial t}=\left[\frac{1}{2} \frac{\partial^{2}}{\partial x^{2}}+v_{\mathrm{eff}}^{1}-i \frac{\partial L_{1}^{\nu}}{\partial p}(x, 0) \frac{\partial}{\partial x}+L_{2}^{\nu}(x, 0)\right] \psi .
$$

4. Наконец, можно рассмотреть случай, когда $\mu \gg h$ или, в размерных переменных, $\lambda_{\|} \ll \lambda_{\perp} \ll l_{0}$. Этот случай естественно назвать “ультракоротким" режимом. Для того чтобы уравнение (2.9) имело смысл, необходимо наложить дополнительные условия на поведение функций $L_{j}^{\nu}$ по переменной $p$. Ограничимся случаем, когда $L_{j}^{\nu}-$ полиномы не более второй степени по $p$. Тогда нетрудно показать, что к уравнению (2.9) может быть применено квазиклассическоеприближение для любых $\mu \gg h$. Этого, однако, оказывается недостаточно для того, чтобы по функции $\psi(x, t)$ с помощью формулы (2.6) можно было восстановить асимптотическое решение исходного уравнения Шредингера в волноводе. Например, если первая поправка $\chi_{1}^{\nu}$ в разложении символа сплетающего операторе $\hat{\chi}^{\nu}$ в формуле (2.7) линейно зависит от $p, \mu \geqslant h^{2}$ и в качестве $\psi$ выбрать быстроосциллируюшую функцию типа (2.11), то функция $\mu \hat{\chi}_{1} \psi$ оказывается не малой. В этом смысле разложение оператора $\hat{\chi}$ по степеням $\mu$ не будет асимптотическим. По этой причине, как мы увидим ниже, для искривленных нанотрубок ультракороткоеприближение работает в случае, когда $h^{2} \ll \mu \ll h$. Можно еше отметить, что в ультракоротком случае реальный эффективный потенциал $v_{\text {eff }}^{1}$ оказывается таким малым, что он может быть перемещен в поправку $L_{1}^{\nu}$ или даже вообще опущен. Тогда эффективный квазиклассический гамильтониан совпадает с гамильтонианом свободной частицы, и в этом случае квазиклассика есть просто борновское приближение.

ЗАмЕчАниЕ 2. Следует иметь в виду, что приведенная классификация (по параметрам $\mu$ и $h$ ) в конкретных расчетах требует уточнения, связанного с величинами как внешних полей, так и поля кристалла и соотношений между ними. При этом, конечно, поправки можно включать и в старшую часть символа (в классический гамильтониан), что, однако, может неоправданно усложнить процедуру построения асимптотических решений. С другой стороны, как мы уже отмечали, в реальной ситуации каждый параметр есть конкретное малое число, поэтому дальнейшая детализация зависимости эффективного гамильтониана и поправок к нему от соотношения между параметрами $\mu$ и $h$ будет носить скорее академический, нежели практический характер. Учитьвая это соображение, с математической точки зрения удобно зафиксировать связи между параметрами $\mu$ и $h$, считая для режима $1 h=\mu$, для режима $2 h=\sqrt{\mu}$, для режима $3 h=1$, 
для режима $4 h=\mu^{3 / 2}$, и включать "оставшиеся" куски из соотношений между $\mu$ и $h$ в коэффициенты уравнения (напряженности электрических и магнитных полей, кривизну и т.д.).

2.10. Число членов в сплетающем операторе $\hat{\chi}^{\nu}$. Будем считать, что мы используем безразмерное время $t$, согласованное с квазиклассическим параметром $h$ (см. п. 2.7). Предположим, что мы сохранили в разложении символов операторов $\hat{\chi}^{\nu}$ и $\hat{L}^{\nu}$ $N$ членов, задаваемых коэффициентами $\chi_{j}^{\nu}$ и $L_{j}^{\nu}$. Кроме того, предположим, что мы построили функцию $\psi^{\nu}$, удовлетворяюшую редуцированному уравнению (2.9) с точностью до некоторой невязки $f$. Из формул приложения 2 следует, что при соответствуюшем определении $\chi_{j}^{\nu}$ и $L_{j}^{\nu}$ подстановка функции $\Psi=\hat{\chi}^{\nu} \psi^{\nu}$ в исходное уравнение дает следующий результат:

$$
i h \frac{\partial \Psi}{\partial t}-\frac{h^{2}}{\mu^{2}} \widehat{\mathcal{H}} \Psi=h^{2} \mu^{N-1} \widehat{F} \psi^{\nu}+\hat{\chi}^{\nu} f .
$$

Здесь $\widehat{F}$, вообше говоря, псевдодифференциальный оператор, не изменяюший (как и оператор $\hat{\chi}^{\nu}$ ) порядка функций $\psi^{\nu}$ по параметру $h$, если функции осциллируют с характерной длиной волны, не меньшей $h$. Применение оценки $(2.10)$ к этому уравнению немедленно приводит к следуюшему выводу: функция $\Psi$ будет отличаться от точного решения на величину порядка $O\left(h^{\delta}\right), \delta>0$, при $h \ll 1$ или $O(\mu)$ при $h=1$, если $N \geqslant 1$ и невязка $f$ равна $O\left(h^{\delta+1}\right)$ при $h \ll 1$ или $O(\mu)$ при $h=1$. Таким образом, минимально разумное число членов в разложении оператора $\hat{\chi}^{\nu}$ при построении квазиклассических асимптотик равно двум (т.е. нужно учитывать нулевой и первый члены). Если же нас интересует длинноволновое приближение (т.е. случай $h=1$ ), то очевидно, что в разложении $\hat{\chi}^{\nu}$ следует учитывать три члена разложения (т.е. $N=2$ ). При этом редуцированное уравнение (2.9) достаточно решить с точностью до $O(\mu)$.

Задача вычисления символов операторов $\hat{\chi}^{\nu}$ и $\hat{L}^{\nu}$ близка к задачам теории возмушения операторов с дискретным спектром (матриц, в частности), причем функция $L^{\nu}$ аналогична собственному значению, а $\chi^{\nu}$ - собственной функции. Члены разложения символов $\chi^{\nu}$ и $L^{\nu}$ вычисляются последовательно, но явное вычисление $L_{j}^{\nu}$ предшествует вычислению $\chi_{j}^{\nu}$ и опирается лишь на факт сушествования $\chi_{j}^{\nu}$. С другой стороны, главный член асимптотики уже определяется $\chi_{0}^{\nu}$ (и, естественно, функцией $\psi^{\nu}$ ). Таким образом, для построения квазиклассических асимптотик в минимально разумном приближении явные формулы нужны лишь для $L_{0}^{\nu}, L_{1}^{\nu}$ и $\chi_{0}^{\nu}$, а для длинноволновых асимптотик - для $L_{0}^{\nu}, L_{1}^{\nu}, L_{2}^{\nu}$ и $\chi_{0}^{\nu}, \chi_{1}^{\nu}$. При этом, как мы уже обсуждали в п. 2.9 , в случаях средневолнового и длинноволнового приближения $(h \gg \mu)$ при вычислении $L_{1}^{\nu}$ достаточно ограничиться значением этой функции и ее первой производной при $p=0$, а функцию $L_{2}^{\nu}$ достаточно вычислить при $p=0$. Этот факт играет важную роль для получения явных формул. Необходимо подчеркнуть, что хотя слагаемые $\mu \hat{\chi}_{1}^{\nu} \psi^{\nu}$ при $h \ll 1$ и $\left(\mu \hat{\chi}_{1}^{\nu}+\mu^{2} \hat{\chi}_{2}^{\nu}\right) \psi^{\nu}$ при $h \sim 1$ оказываются лишь поправками к главному члену асимптотического разложения $\hat{\chi}_{0} \psi^{\nu}$, тем не менее для его правильного описания существование таких поправок должно быть гарантировано, и просто подстановка функции $\hat{\chi}_{0}^{\nu} \psi^{\nu}$ в исходное уравнение не даст невязки, требуемой для установления малости между $\hat{\chi}_{0}^{\nu} \psi^{\nu}$ 
и точным решением. Здесь имеется отличие от стандартного квазиклассического приближения для скалярного уравнением Шредингера: в последнем случае подстановка главного члена асимптотики в исходное уравнение сразу дает требуемую малую невязку $O\left(h^{2}\right)$.

2.11. Существенная часть квантового гамильтониана для продольного движения. Таким образом, согласно проведенному в предыдущих пунктах анализу, мы приходим к выводу, что во всех рассмотренных случаях 1-4 главный член асимптотического разложения $\psi^{\nu}$ (а вместе с ним и $\Psi$ ) будет полностью определяться (иногда с избытком точности) квантовым гамильтонианом

$$
\hat{L}_{h}^{\nu}=\frac{1}{2}\left(-i h \frac{\partial}{\partial x}\right)^{2}+v_{\text {eff }}^{1}+\frac{h^{2}}{\mu}\left[L_{1}^{\nu}\left(\frac{2}{x}, \frac{\mu}{h}\left(-i h \frac{\partial}{\partial x}\right)\right)+\mu L_{2}^{\nu}(x, 0)\right] .
$$

Его естественно назвать существенной частью гамильтониана продольного движсения на $\nu$-й подзоне размерного квантования.

2.12. Обобщение формулы “операторного разделения" для нескольких поперечных и продольных переменных и векторных уравнений. Если рассмотреть уравнение Шредингера не в полосе, а в цилиндре, то оператор $\mathcal{L}$, действуюший в пространстве функций, зависяших от переменной $y$, следует заменить на оператор, действуюший на функции от двух переменных, например, $y_{1}$ и $y_{2}$. Теперь спектр оператора $\mathcal{L}$ уже может быть вырожденным. Вообше говоря, кратность вырождения

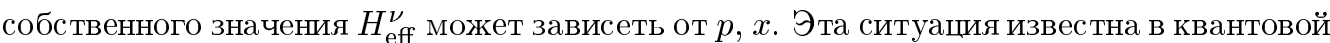
механике как эффект "пересечения термов" (см., например, [19], [23]). Мы ограничимся случаем, ког да кратность вырождения рассматриваемого эффективного гамильтониана не зависит от $(p, x)$ и равна, скажем, $k$. Обозначим соответствующие собственные функции $\chi^{\nu, l}, l=1,2, \ldots, k$. Каждая такая функция порождает оператор $\hat{\chi}^{\nu, l}$ и функцию $\psi^{\nu, l}$. В случае точного разделения переменных взаимодействие между состояниями, описываемыми различными функциями $\psi^{\nu, l}$, отсутствует, и для определения каждой из функций можно использовать скалярные уравнения. В противном случае для их определения мы имеем, вообше говоря, систему. Обозначим через $\psi^{\nu}$ вектор-столбец, состоящий из $\psi^{\nu, l}$, и будем понимать под оператором $\hat{L}^{\nu}$ в уравнении (2.4) матричный оператор $\hat{L}^{\nu}=L^{\nu}(-i \mu \partial / \partial x, x, \mu), L^{\nu}(p, x, \mu)=H_{\text {eff }}^{\nu} E_{k}+\mu L_{1}^{\nu}+\cdots$ с диагональным главным членом $\widehat{H}_{\text {eff }} E_{k}$. Здесь $E_{k}-(k \times k)$-единичная матрица, а $L_{1}^{\nu}, \ldots-(k \times k)$-матричнозначные функции, зависяшие от $p, x$. Теперь мы имеем $k$-мерное векторное уравнение $(2.4)$ для вектор-функции $\psi^{\nu}$. Взаимодействие между функциями $\psi^{\nu, l}$ определяется поправками $L_{1}^{\nu}, L_{2}^{\nu}, \ldots$. Формула операторного разделения переменных снова имеет вид (2.6), если мы понимаем под $\hat{\chi}^{\nu}$ вектор-строку, состояшую из операторов $\hat{\chi}^{\nu, l}, l=1,2, \ldots, k$. И, наконец, мы можем рассматривать $s$-векторное уравнение (например, уравнения Паули или Дирака) вместо исходного скалярного уравнения. Мы можем повторить предыдушие соображения; разница заключается в том, что теперь имеется векторная спектральная задача для эффективных гамильтонианов и функций $\chi^{\nu, l}$, и нужно рассматривать $\hat{\chi}^{\nu}$ как $(k \times s)$-матрицу, элементами которой являются операторы. Именно такая ситуация и имеет место в рассматриваемых нами задачах, порожденных оператором $\widehat{\mathcal{H}}$ вида (1.1) в нанотрубках. 
Рассматриваемое приближение соответствует переходу к представлению, в котором матрица исходного оператора приведена к “почти” блочному виду с малыми межблочными элементами, и учету этих элементов во взаимодействуюших амплитудах. Блоки соответствуют вырожденным адибатическим термам. Таким образом, мы учитываем взаимодействие внутри терма и считаем, что взаимодействие между непересекающимися термами экспоненциально (по параметру $\mu$ ) мало. С “классической” точки зрения вырождение терма соответствует наличию резонансов некоторого типа.

2.13. Квазиклассическое расщепление адиабатических термов. При $h \gg$ $\mu$ поправка $L_{1}(x, 0)$ может изменить эффективные гамильтонианы. Такое изменение оказывается особенно существенным в том случае, когда терм вырожден или (и) когда исходная задача - векторная (т.е. ког да исходный квантовый гамильтониан матричный). Остановимся на этой ситуации. В силу самосопряженности исходной задачи матрица $L_{1}(x, 0)$ эрмитова. Предположим, что все ее собственные значения $\lambda_{1}(x), \ldots, \lambda_{k}(x)$ различны и вместе с собственными векторами гладко зависят от $x$. Кроме того, для того чтобы не заниматься перенормировкой энергии, будем считать для простоты, что $\lambda_{j}(0)=0$. В силу вырождения адиабатического терма имеется большой произвол в выборе вектор-функций $\chi_{j}^{\nu}, j=1, \ldots, k$, причем изменение в их выборе естественно приводит к изменению матрицы $L_{1}^{\nu}$. Выберем вектор-функции $\chi_{j}^{\nu}, j=1, \ldots, k$, так, чтобы матрица $L_{1}^{\nu}(x, 0)$ была диагональной. Если $1 \gg h \gg \mu$ (“средневолновое" приближение), то в квазиклассический гамильтониан следует добавить выражения $\left(h^{2} / \mu\right) \lambda_{j}(x)$. Проводя рассуждения, аналогичные п. 2.6, приходим к выводу, что параметр $h$ должен удовлетворять неравенству $\sqrt{\mu} \geqslant h \gg \mu$. Мы видим, что квазиклассические гамильтонианы (термы) $(1 / 2)\left(p^{h}\right)^{2}+v_{\text {eff }}^{1}+\left(h^{2} / \mu\right) \lambda_{j}$ становятся различными для разных $j$, и тем самым происходит "квазиклассическое" расшепление "адиабатических" термов. Величина этого расщепления зависит от числа $h^{2} / \mu$ и достигает наибольшей величины при $h \sim \sqrt{\mu}$. В первом приближении по параметру $h^{2} / \mu$ состояния, отвечающие разным квазиклассическим гамильтонианам, не взаимодействуют. Тем самым внутри адиабатического терма мы можем асимптотически диагонализовать систему для вектор-функции $\psi^{\nu}$. Хотя этот асимптотический факт оказывается справедливым для любых значений $h$ из указанного выше интервала, тем не менее понятно, что при значениях $h$, близких к $\mu$, следуюшие члены разложения могут быть настолько большими, что указанное представление о такой асимптотической “диагонализации” теряет смысл. Поэтому ситуацию с $h$, близким к $\mu$, следует рассматривать как ситуацию с вырожденным термом, и слагаемые $\left(h^{2} / \mu\right) \lambda_{j}$ более естественно оставить в исходной поправке $\left(h^{2} / \mu\right) L_{1}^{\nu}$, что позволяет более адекватно учитывать взаимодействие между состояниями внутри адиабатического терма. Последнее рассуждение относится к случаю матричной поправки $L_{1}^{\nu}$. В скалярной ситуации, как будет ясно из дальнейшего, слагаемое $\left(h^{2} / \mu\right) L_{1}^{\nu}(x, 0)$ всегда можно включить в эффективный гамильтониан. Более подробно мы обсудим затронутые в этом пункте вопросы при построении конкретных асимптотических решений в части II этой работы. Подчеркнем только, что все указанные в этом и предыдущем пунктах явления полностью определяются существенной частью матричного аналога гамильтониана продольного движения на $\nu$-й подзоне размерного квантования (см. п. 2.10). 
Цель последующих разделов настояшей работы состоит в явном описании сушественной части оператора $\widehat{\mathcal{H}}$ вида (1.1) в нанотрубках.

\section{3. КВАНТОВЫЙ ГАМИЛЬТОНИАН В ТОНКОЙ ТРУБКЕ В БЕЗРАЗМЕРНЫХ ПЕРЕМЕННЫХ}

3.1. Безразмерные переменные и поля. Вернемся к квантово-механическим уравнениям, задаваемым оператором (1.1) в тонкой трубке. Сначала перепишем уравнения в подходящих безразмерных координатах. Под характерным "продольным" размером $l_{0}$ мы будем подразумевать длину трубки, характерный радиус трубки обозначим через $d_{0}$, причем, как и в модельном примере (раздел 2 ), мы будем предполагать, что радиус кривизны $\sim l_{0}$. Перейдем от декартовых координат $\mathbf{r}$ в гамильтониане $(1.1)$ к безразмерным координатам $\mathbf{r}^{\prime}=\mathbf{r} / l_{0}$. Введем "магнитную длину" $l_{\mathrm{M}}=\sqrt{\hbar c /(e|\mathbf{H}|)}$, квант магнитного потока $\Phi_{0}=2 \pi \hbar c / e$, безразмерное магнитное поле как "число квантов магнитного потока через характерную площадь $l_{0} d_{0} ": \mathbf{H}^{\prime}=2 \pi l_{0} d_{0} \mathbf{H} / \Phi_{0}, \quad\left|\mathbf{H}^{\prime}\right|=$ $|\mathbf{H}| l_{0} d_{0} / l_{\mathrm{M}}^{2}$, и безразмерный векторный потенциал $\mathbf{A}^{\prime}$. Характерная магнитная длина, соответствуюшая напряженности магнитного поля $10^{4} \Gamma$ с (1 Тл), определяется следующим образом: $l_{\mathrm{M}}=\sqrt{\hbar c / e|\mathbf{H}|}=\sqrt{e / \alpha_{0}|\mathbf{H}|} \simeq 20 \mathrm{Hм}, \alpha_{0}=e^{2} / \hbar c=1 / 137$. Таким образом, для нанотрубок с длиной в несколько сот нанометров получаем $l_{0} d_{0} / l_{\mathrm{M}}^{2} \sim 1$.

Определим безразмерные потенциалы $v_{\mathrm{int}}^{\prime}=v_{\mathrm{int}} / \varepsilon_{\perp}, v_{\mathrm{ext}}^{\prime}=v_{\mathrm{ext}} / \varepsilon_{\perp}$ и безразмерный параметр, характеризуюший спин-орбитальное взаимодействие $\alpha^{\prime}=\hbar \alpha / d_{0}^{2}\left(\varepsilon_{\perp}-\right.$ характерная поперечная энергия, см. введение). Штрихи в дальнейшем будем опускать. Квантовый гамильтониан, зависящий от введенных выше безразмерных величин и отнесенный к $\varepsilon_{\perp}$, примет вид

$$
\widehat{\mathcal{H}}=\frac{1}{2}(-i \mu \nabla-\mathbf{A})^{2}+v_{\text {int }}(\mathbf{r})+v_{\text {ext }}(\mathbf{r}, t)+\frac{\mu}{2}\langle\boldsymbol{\sigma}, \mathbf{H}\rangle+\widehat{\mathcal{H}}_{\mathrm{SO}},
$$

где $\widehat{\mathcal{H}}_{\mathrm{SO}}=\alpha\left\langle\boldsymbol{\sigma},\left[\nabla v_{\mathrm{int}},-i \mu \nabla-\mathbf{A}\right]\right\rangle$. Безразмерный параметр $\alpha$, как отмечалось вьше, зависит от типа кристалла. Так, например, для кристалла InAs $\alpha=0.145 \varepsilon_{\perp} / E_{g}[5]$. Если положить $\varepsilon_{\perp}=0.1$ эВ, то параметр $\alpha=0.0354 \sim \mu[7]$.

3.2. Координаты, связанные с осью трубки (рис. 1). Будем считать, что ось трубки - кривая $\gamma$ - задана уравнением $\mathbf{r}=\mathbf{R}(x), \mathbf{r} \in \mathbb{R}^{3}$, где $\mathbf{R}(x)$ - гладкая вектор-функция, $x \in \mathbb{R}$ - натуральный параметр на $\gamma$ (длина трубки, отсчитанная от некоторой фиксированной точки $\left.x^{*}\right),\left|\partial_{x} \mathbf{R}(x)\right|=1, \partial_{x}=\partial / \partial x$. В случае, если $\left|\partial_{x}^{2} \mathbf{R}\right| \neq 0$, определен трехгранник Френе. Кривизна $k(x)=\left|\partial_{x}^{2} \mathbf{R}\right|$ и кручение $\varkappa(x)$ кривой $\gamma$ связаны с трехгранником $\Phi$ рене $\left\{\partial_{x} \mathbf{R}, \mathbf{n}=\partial_{x}^{2} \mathbf{R} /\left|\partial_{x}^{2} \mathbf{R}\right|, \mathbf{b}=\left[\partial_{x} \mathbf{R}, \mathbf{n}\right]\right\}$ в каждой точке $x$ формулами $\partial_{x} \mathbf{n}=-\varkappa \mathbf{b}-k \partial_{x} \mathbf{R}, \partial_{x} \mathbf{b}=\varkappa \mathbf{n}$.

Обозначим через П $(x)$ плоскость, ортогональную оси трубки в точке $\mathbf{R}(x)$; сечение трубки этой плоскостью (область в П $(x)$ ) обозначим $\Omega(x)$, границу $\Omega(x)-$ как $\partial \Omega(x)$. Тогда трубка получается в результате объединения по $x$ областей $\Omega(x)$, а ее граница - кривых $\partial \Omega(x)$. Введем внутри трубки новые координаты $\left(x, y_{1}, y_{2}\right)$ по формуле $\mathbf{r}=\mathbf{R}(x)+\mathbf{y}(x), \mathbf{y}(x)=y_{1} \mathbf{n}_{1}(x)+y_{2} \mathbf{n}_{2}(x)$, где $\left\{\mathbf{n}_{1}(x), \mathbf{n}_{2}(x)\right\}$ - базис в плоскости $\Pi(x)$. 
Если положить $\mathbf{n}_{1}=\mathbf{n}, \mathbf{n}_{2}=\mathbf{b}$, то введенные таким образом координаты будут неортогональными. Удобнее ввести ортогональные координаты (см. [17], [24]). Пусть сначала $\left\{\mathbf{n}_{1}(x), \mathbf{n}_{2}(x)\right\}$ - некоторый ортонормированный базис на плоскости П $(x)$, гладко зависящий от $x$ (не совпадаюший, вообше говоря, с $\mathbf{n}, \mathbf{b}$ ); $\theta(x)$ - угол между векторами $\mathbf{n}$ и $\mathbf{n}_{1}$. Тогда наряду с кручением $\varkappa$ можно вычислить "эффективное кручение" $\varkappa_{\text {eff }}=$ $-\left\langle\partial_{x} \mathbf{n}_{1}, \mathbf{n}_{2}\right\rangle=\varkappa-\partial_{x} \theta$. Выбирая угол $\theta(x)$ (а тем самым и векторы $\left.\left\{\mathbf{n}_{1}(x), \mathbf{n}_{2}(x)\right\}\right)$ так,

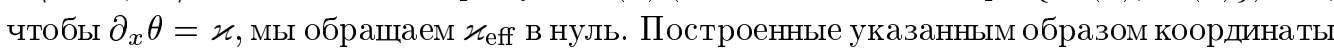
(в окрестности трубки, где они определены) являются ортогональными. Компоненты метрического тензора $g_{i j}, i, j=\left\{x, y_{1}, y_{2}\right\}$, в этих координатах определяются следуюшим образом: $g_{00}=G=(1-k\langle\mathbf{y}, \mathbf{n}\rangle)^{2}, g_{11}=g_{22}=1, g_{i j}=0, i \neq j$. Всюду ниже мы пользуемся именно такими координатами. При этом получаемые в дальнейшем формулы будут справедливы для случая прямой оси, если положить $k(x)=0, \varkappa(x)=0$. В случае оси трубки с ненулевой кривизной $y_{1}$ и $y_{2}$ образуют координаты лишш для таких значений, для которых $1-k\langle\mathbf{y}, \mathbf{n}\rangle>0$. Из предположений для кривизны оси трубки, приводимых ниже, следует, что внутри трубки такие координаты определены.

3.3. Моделирование области конфайнмента. Спектральная задача для поперечных мод. Рассматривая модельный пример, мы уже обсуждали основные принципы моделирования области конфайнмента квазичастишы, а также тот факт, что область, ограниченная “жесткими стенками” (моделируемыми условиями Дирихле для волновой функции на боковой поверхности), может, вообще говоря, не совпадать с цилиндрообразной поверхностью, на которой расположен ионный остов физической трубки (см. рис. 1). С формальной математической точки зрения после введения соответствующего кристаллического потенциалав области $\Omega$, быстро растущего у поверхности $\partial \Omega$, мы можем "забыть" как об исходной физической трубке, так и об условиях Дирихле на $\partial \Omega$, считая, что волновые функции так быстро убывают при отдалении $y$ от ионного остова, что условия Дирихле практически выполнены автоматически. Это соображение, позволяющее рассматривать $y$ как координаты на всей плоскости П $(x)$, дает возможность значительно упростить многие формулы без существенной потери точности. С другой стороны, с математической точки зрения, для того чтобы не беспокоиться о корректности введенных вьше координат при больших значениях $|y|$, "мягкие стенки" удобно дополнять “жесткими стенками”, считая, что на боковой границе $\partial \Omega$ волновые функции удовлетворяет условиям Дирихле. Так мы и будем поступать в дальнейшем.

Будем рассматривать трубки, сечение которых плоскостью П $(x)$ врашается относительно базиса $\left\{\mathbf{n}_{1}(x), \mathbf{n}_{2}(x)\right\}$ и одновременно растягивается в плоскости $x=$ const относительно точки $\mathbf{R}(x)$. Киральность трубки зададим следуюшим образом: зафиксируем сечение $\Omega\left(x^{*}\right)$ при некотором $x^{*} \in \gamma$, считая, что сечение $\Omega(x)$ получается из $\Omega\left(x^{*}\right)$ поворотом на "угол внутреннего кручения" $\Phi(x)$ относительно базиса $\left\{\mathbf{n}_{1}(x), \mathbf{n}_{2}(x)\right\}$ и растяжением в $D(x)$ раз (рис. 1 ). Сжатие и растяжение сечения моделирует, например, ситуацию, когда трубка деформируется силами Ван дер Ваальса [25].

Обсудим теперь вопросы, связанные с выбором модельного потенциала. Напомним, что в нашей модели на "геометрию" сечения трубки потенциал конфайнмента может влиять даже в большей степени, чем "жесткие" стенки. Рассмотрим следуюший вид модельного потенциала, позволяющего моделировать "круговые" и "эллиптические" 
сечения трубки, обладающей киральностью:

$v_{\text {int }}(x, y)=v_{\text {int }}\left(x^{*}, \frac{\boldsymbol{\Phi}(x)^{-1} \mathbf{y}(x)}{D(x)}\right), \quad v_{\text {int }}\left(x^{*}, y\right)=\left[\left(\frac{y_{1}}{a}\right)^{2}+\left(\frac{y_{2}}{b}\right)^{2}\right]^{m}, \quad a, b, m>0$

где $D(x)>0$ - гладкая функция $x, \boldsymbol{\Phi}(x)$ - матрица поворота сечения трубки на угол $\Phi(x)$. Аналогично модельному примеру, энергия $\nu$-й поперечной моды в точке $x$ определяется как собственное значение $\varepsilon_{\perp}^{\nu}(x)$ стационарного уравнения Шредингера

$$
\left(-\frac{\Delta_{y}}{2}+v_{\text {int }}(x, y)\right) w_{\nu}=\varepsilon_{\perp}^{\nu}(x) w_{\nu}, \quad\left\|w_{\nu}\right\|(x)=1
$$

Переходя от переменных $y=\left(y_{1}, y_{2}\right)$ к новым переменным $y^{\prime}=\left(y_{1}^{\prime}, y_{2}^{\prime}\right)$, определенным равенством $y=D^{\gamma} y^{\prime}, \gamma=m /(m+1)$, находим, что $\varepsilon_{\perp}^{\nu}(x)=D(x)^{-2 \gamma} \varepsilon_{\perp}^{\nu}(0)$. Легко видеть, что дисперсия $d(x)$ по координатам $y$ в состоянии $w_{n}$ зависит от $x$ согласно соотношению $d(x)=D(x)^{-\gamma} d(0)$. Естественно считать, что $d(x)$ пропорииональна линейным размерам сечения трубки. Тогда $D^{\gamma}$ - "мягкий" коэффициент растяжения сечения, а $\gamma-$ коэффишиент "жесткости” стенок. Зависимость энергии от $x$ можно представить в виде $\varepsilon_{\perp}^{\nu}(x)=\varepsilon_{\perp}^{\nu}(0) d(0)^{2} / d(x)^{2}$. При $m \rightarrow \infty$ потенциал (3.2) исчезает внутри области, а вне ее стремится к $\infty$; коэффициент $\gamma \rightarrow 1, d(x) \rightarrow D(x)^{-1} d(0)$. В пределе мы приходим к модели “пустого цилиндра": $v_{\text {int }}\left(x^{*}, y\right)=0$ при $\left(y_{1} / a\right)^{2}+\left(y_{2} / b\right)^{2} \leqslant 1$, и $v_{\text {int }}\left(x^{*}, y\right)=\infty$ при $\left(y_{1} / a\right)^{2}+\left(y_{2} / b\right)^{2}>1$, для которой $D(x)$ - коэффициент растяжения (гомотетии).

Область $\Omega(x)$ можно задать как неодносвязную, например, в виде кругового или эллиптического кольца. Адекватность такого представления зависит от вида кристаллического потенциала в конкретной нанотрубке. Если область $\Omega(x)$ односвязна, то в физической литературе такую нанотрубку называют “квантовой проволокой” [7].

Характерный размер сечения трубки в безразмерных координатах, очевидно, имеет порядок $\mu$, поэтому, как и в модельном примере, нам удобно перейти к растянутым переменным, заменив $y \rightarrow \mu y$. Тогда исходный оператор $\widehat{\mathcal{H}}$ примет вид $\widehat{\mathcal{H}}=\mathcal{H}(-i \mu \partial / \partial x$, $\stackrel{2}{x},-i \partial / \partial y, y, t, \mu)$, где индексы сверху обозначают порядок действия некоммутируюших операторов $x$ и $-i \mu \partial / \partial x$ (выбор упорядочения некоммутирующих операторов $y$ и $-i \partial / \partial y$ для последующих выкладок несушественен). Оператор $\mathcal{H}(p, x,-i \partial / \partial y, y, t, \mu)$, действуюший (при фиксированных $x, p, t)$ в пространстве функций, зависящих от поперечных координат $y=\left(y_{1}, y_{2}\right)$ (быстрых переменных), есть (см. п. 2.4) операторнозначный символ оператора $\widehat{\mathcal{H}}$ [13]. В соответствии с рассуждениями, приведенными в предыдушем разделе, для дальнейшего нам важны только первые три члена адиабатического разложения оператора $\mathcal{H}$ по параметру $\mu$ :

$$
\begin{aligned}
\mathcal{H}\left(p, x,-i \frac{\partial}{\partial y}, y, t, \mu\right)= & \mathcal{H}_{0}\left(p, x,-i \frac{\partial}{\partial y}, y, t\right)+\mu \mathcal{H}_{1}\left(p, x,-i \frac{\partial}{\partial y}, y, t\right)+ \\
& +\mu^{2} \mathcal{H}_{2}\left(p, x,-i \frac{\partial}{\partial y}, y, t\right)+O\left(\mu^{3}\right)
\end{aligned}
$$

5 Теоретическая и математическая физика, т. 141, № 2, 2004 г. 
Приведем явные формулы для операторнозначных символов $\mathcal{H}_{j}$ “ $\mu$-дифференциальных" операторов $\widehat{\mathcal{H}}_{j}, j=0,1$ (вывод этих формул см. в приложении 1):

$$
\begin{aligned}
\mathcal{H}_{0}= & \frac{\mathcal{P}_{0}^{2}}{2}+v_{\text {ext }}(\mathbf{R}(x), t)+\sum_{j=1}^{2} \frac{\widehat{\mathcal{P}}_{j}^{2}}{2}+v_{\text {int }}(x, y), \\
\mathcal{H}_{1}= & -\frac{1}{2}\left\langle\partial_{x} \mathbf{R}, \mathbf{H}\right\rangle \hat{l}+\frac{i k}{2}\left\langle\mathbf{n}, \mathbf{A}_{0}\right\rangle+\left(k\langle\mathbf{y}, \mathbf{n}\rangle \mathcal{P}_{0}-\frac{1}{2}\left\langle\mathbf{y}_{\perp}, \mathbf{H}\right\rangle\right) p+ \\
& +\left\langle\nabla v_{\text {ext }}(\mathbf{R}(x), t)+\frac{1}{2}\left[\mathbf{A}_{0}, \mathbf{H}\right], \mathbf{y}\right\rangle-\frac{1}{2}\langle\boldsymbol{\sigma}, \mathbf{H}\rangle+\mu^{-1} \alpha\langle\boldsymbol{\sigma}, \widehat{\mathbf{M}}\rangle .
\end{aligned}
$$

Здесь введены обозначения

$$
\begin{gathered}
\mathcal{P}_{0}=p-\left\langle\partial_{x} \mathbf{R}, \mathbf{A}_{0}\right\rangle, \quad \widehat{\mathcal{P}}_{j}=-i \frac{\partial}{\partial y_{j}}-\left\langle\mathbf{n}_{j}, \mathbf{A}_{0}\right\rangle, \quad j=1,2, \quad \hat{l}=i\left(y_{2} \frac{\partial}{\partial y_{1}}-y_{1} \frac{\partial}{\partial y_{2}}\right), \\
\mathbf{y}_{\perp}=\left[\mathbf{y}, \partial_{x} \mathbf{R}\right]=y_{1} \mathbf{n}_{2}-y_{2} \mathbf{n}_{1}, \quad \mathbf{A}_{0}=\frac{1}{2}[\mathbf{H}(t), \mathbf{R}(x)], \\
\widehat{\mathbf{M}}=\partial_{x} \mathbf{R}\left(\frac{\partial v_{\text {int }}}{\partial y_{1}} \widehat{\mathcal{P}}_{2}-\frac{\partial v_{\text {int }}}{\partial y_{2}} \widehat{\mathcal{P}}_{1}\right)+\mathbf{n}_{1} \frac{\partial v_{\text {int }}}{\partial y_{2}} \mathcal{P}_{0}-\mathbf{n}_{2} \frac{\partial v_{\text {int }}}{\partial y_{1}} \mathcal{P}_{0} .
\end{gathered}
$$

Для символа $\mathcal{H}_{2}$ приведем только его геометрическую часть $\mathcal{G}(x)=-k^{2} / 8$, “остаток" $\mathcal{H}_{2}-\mathcal{G}$ есть полином по импульсу $\mathcal{P}_{0}$ и компонентам магнитного поля $\mathbf{H}(t)$ с нулевым свободным членом и гладко зависяшими от $(x, y)$ коэффициентами. Из дальнейшего будет видно, что явный вид этого “остатка" нам не понадобится.

Сохраняя пока время $t$ размерным, приведем нестационарное уравнение Паули в нанотрубке во введенных безразмерных пространственных координатах:

$$
i T \mu \frac{\partial}{\partial t} \Psi(x, y, t, \mu)=\mathcal{H}\left(-i \mu \frac{\partial}{\partial x}, \stackrel{2}{x},-i \frac{\partial}{\partial y}, y, t, h\right) \Psi(x, y, t, \mu), \quad T=\frac{m d l_{0}}{\hbar}
$$

\section{4. АДИАБАТИЧЕСКИЙ И КВАЗИКЛАССИЧЕСКИЕ ГАМИЛЬТОНИАНЫ НА ПОДЗОНЕ РАЗМЕРНОГО КВАНТОВАНИЯ}

4.1. Редукция к уравнениям на оси трубки и адиабатический гамильтониан. Как мы уже отмечали вьше, уравнение (3.6) относится к классу уравнений с "операторнозначным символом". Его асимптотические решения для перечисленных во введении задач мы получаем с помошью предложенной в работах [19] схемы (см. приложение 2), представляющей собой модификацию общего подхода [13] и изложенной в разделе 2 на модельном примере. Согласно этой схеме процедура построения нахождения решений разделяется на два этапа: 1) асимптотическую редукцию исходного уравнения к набору пространственно-одномерных уравнений; 2) построение асимптотических решений этих редуцированных одномерных уравнений. Специфика рассматриваемой задачи, в отличие от модельного примера, заключается, во-первых, в наличии магнитного поля. Во-вторых, двумерное уравнение (3.3) при определенных условиях для вида 
кристаллического потенциала $v_{\text {int }}(x, y)$ может иметь вырожденный спектр. Кроме того, исходный гамильтониан (3.1) является матричным, так как учитывает наличие спина.

Заметим, что поскольку редуцированное уравнение, аналогичное (2.4), содержит одну пространственную переменную, то в волновой функции естественно сразу выделить множитель $\exp \left(i \int_{x^{*}}^{x}\left\langle\partial_{x} \mathbf{R}, \mathbf{A}_{0}\right\rangle d x / \mu\right)$, что переведет в редуцированных уравнениях удлиненный оператор импульса $\widehat{\mathcal{P}}$ в “короткий” $\hat{p}=-i \mu \partial / \partial x$, но в случае зависяшего от времени $t$ магнитного поля даст поправку $\int_{x^{*}}^{x}\left\langle\partial_{x} \mathbf{R}, \partial \mathbf{A}_{0} / \partial t\right\rangle d x$ к эффективному потенциалу. Далее, в силу того что функция $\Psi$ представляет собою спинор, истинная кратность вырождения терма, определяющего редуцированное уравнение, равна $2 r$, где $r$ кратность вырождения собственного значения $\varepsilon_{\perp}^{\nu}(x)$ задачи (3.3).

С учетом этих замечаний приведем результаты первого шага редукции (соответствующие вычисления см. в приложениях 2,3$)$. Решение $\Psi$ уравнения $(3.6)$ представляется в виде

$$
\Psi(x, y, t, \mu)=\hat{\chi}^{\nu}\left[\exp \left(i \int_{x^{*}}^{x}\left\langle\partial_{x} \mathbf{R}, \mathbf{A}_{0}\right\rangle d x / \mu\right) \psi^{\nu}\right], \quad \hat{\chi}^{\nu}=\chi^{\nu}\left(-i \mu \frac{\partial}{\partial x}, x, y, t, \mu\right)
$$

где $\hat{\chi}^{\nu}$ - матричный (псевдо)дифференциальный оператор с символом $\chi^{\nu}(x, p, y, t, \mu)$, допускаюшим регулярное разложение по $\mu$ в виде $\chi^{\nu}(x, p, y, t, \mu)=\chi_{0}^{\nu}(x, p, y, t, \mu)+$ $\mu \chi_{1}^{\nu}(x, p, y, t, \mu)+\cdots$, а $\psi$ - новая неизвестная вектор-функция с $2 r$ компонентами $\psi^{\nu j}$. Компоненты $\psi^{\nu j}$, вообше говоря, взаимодействуют меж ду собой, и мы считаем, что для $\psi^{\nu}$ справедливо векторное (псевдо) дифференциальное уравнение типа уравнения Шредингера

$$
i T \mu \frac{\partial \psi^{\nu}}{\partial t}=\hat{L}^{\nu} \psi^{\nu}, \quad \hat{L}^{\nu}=L^{\nu}\left(-i \mu \frac{\partial}{\partial x}, 2, x, \mu\right)
$$

где $\hat{L}^{\nu}(-i \mu \stackrel{1}{\partial} / \partial x, \stackrel{2}{x}, \mu)$ - адиабатический гамильтониан, символ которого также разлагается в (асимптотический) ряд типа (2.8). Как показано в разделе 2 , нам понадобится только его существенная часть $L_{0}^{\nu}(p, x)+\mu L_{1}^{\nu}(p, x)+\mu^{2} \mathcal{G}(x)$. Как $L_{j}^{\nu}$, так и $\chi_{j}^{\nu}$ восстанавливаются через решения $\varepsilon_{\perp}^{\nu}$ и $w_{j}^{\nu}$ спектральной задачи $(3.3), \nu$ - номер собственного значения (терма). А именно, $(2 r \times 2)$-матрица $\chi_{0}$ имеет вид

$$
\chi_{0}=\left\|w_{1}^{\nu}, \ldots, w_{r}^{\nu}\right\| \otimes E_{s}=\left\|\begin{array}{ccccc}
w_{1}^{\nu}(x, y) & 0 & \cdots & w_{r}^{\nu}(x, y) & 0 \\
0 & w_{1}^{\nu}(x, y) & \cdots & 0 & w_{r}^{\nu}(x, y)
\end{array}\right\|,
$$

где $\otimes$ - тензорное произведение матрищ, а $E_{s}$ - единичная $(2 \times 2)$-матрица. Матричная функция $\chi_{1}^{\nu}$ находится из уравнения (П.5). Определяемые ниже объекты $H_{\mathrm{eff}}^{\mu}, \Lambda, L_{y}^{\nu}$ и т.д., вообще говоря, также зависят от номера $\nu$ (как и $\left.\chi_{j}, L_{j}^{\nu}, \psi^{\nu}\right)$; иногда мы опускаем эту зависимость для упрошения обозначений. 
Символы $L_{0}^{\nu}$ и $L_{1}^{\nu}$ определяются следующим образом (см. приложение 3$)$ :

$$
\begin{aligned}
L_{0}^{\nu}(p, x) & =H_{\mathrm{eff}}^{\mu} E_{r} \otimes E_{s} \\
H_{\mathrm{eff}}^{\mu} & =\frac{p^{2}}{2}+v_{\mathrm{ext}}(x)+\varepsilon_{\perp}^{\nu}(x)+\int_{0}^{x}\left\langle\partial_{x} \mathbf{R}\left(x^{\prime}\right), \frac{\partial \mathbf{A}_{0}}{\partial t}\left(x^{\prime}, t\right)\right\rangle d x^{\prime} \\
L_{1}^{\nu}(p, x) & =\frac{i k}{2}\left\langle\mathbf{n}, \mathbf{A}_{0}\right\rangle E_{r} \otimes E_{s}+L_{y} \otimes E_{s}+E_{r} \otimes L_{s}+L_{s y}, \quad L_{s}=-\frac{1}{2}\langle\boldsymbol{\sigma}, \mathbf{H}\rangle \\
L_{y}(p, x) & =\left(\left(\partial_{x} \Phi\right) p-\frac{1}{2}\left\langle\partial_{x} \mathbf{R}, \mathbf{H}\right\rangle\right) \Lambda-\left\langle\mathbf{Y}_{\perp}, \mathbf{H}\right\rangle p+\left\langle\mathbf{Y}, \nabla v_{\mathrm{ext}}+\frac{\partial \mathbf{A}_{0}}{\partial t}+k p^{2} \mathbf{n}\right\rangle \\
L_{s y}(p, x) & =\frac{\alpha}{\mu}\left(M^{0} \otimes\left\langle\boldsymbol{\sigma}, \partial_{x} \mathbf{R}\right\rangle+M^{1} \otimes\left\langle\boldsymbol{\sigma}, \mathbf{n}_{1}\right\rangle+M^{2} \otimes\left\langle\boldsymbol{\sigma}, \mathbf{n}_{2}\right\rangle\right)
\end{aligned}
$$

Мы обозначили через $E_{r}$ единичную $(r \times r)$-матрицу, через $\Lambda(x)-(r \times r)$-матрицу момента с элементами $\Lambda_{j j^{\prime}}=\left\langle w_{j}^{\nu}, \hat{l} w_{j^{\prime}}^{\nu}\right\rangle_{y}$, через $M^{j}(x)$ - матрицы размера $(r \times r)$ вида $\left(M^{0}\right)_{j j^{\prime}}=-i\left\langle w_{j}^{\nu},\left(\left(\partial_{1} v_{\text {int }}\right) \partial_{2}-\left(\partial_{2} v_{\text {int }}\right) \partial_{1}\right) w_{j^{\prime}}^{\nu}\right\rangle_{y}, \quad\left(M^{1}\right)_{j j^{\prime}}=\left\langle w_{j}^{\nu},\left(\partial_{2} v_{\text {int }}\right) w_{j^{\prime}}^{\nu}\right\rangle_{y} p$, $\left(M^{2}\right)_{j j^{\prime}}=-\left\langle w_{j}^{\nu},\left(\partial_{1} v_{\text {int }}\right) w_{j^{\prime}}^{\nu}\right\rangle_{y} p$, где $\partial_{i}=\partial / \partial y_{i}$, и через $\mathbf{Y}(x)=Y_{1} \mathbf{n}_{1}+Y_{2} \mathbf{n}_{2}, \mathbf{Y}_{\perp}(x)=$ $Y_{2} \mathbf{n}_{1}-Y_{1} \mathbf{n}_{2}$ - трехмерные "векторы", компоненты которых суть “дипольные" $(2 \times 2)$-матрицы $\left(Y_{i}\right)_{j j^{\prime}}(x)=\left\langle w_{j}^{\nu}, y_{i} w_{j^{\prime}}^{\nu}\right\rangle_{y}, i=1,2$. Как и выше, $\langle\cdot, \cdot\rangle_{y}$ означает интегрирование по переменным $y$. Символ $L_{2}^{\nu}(p, x)$ оказывается сушественно более сложным; нам, однако, нужна только его часть - так называемый “геометрический потенциал” $\mathcal{G}(x)=$ $-\left(k^{2}(x) / 8\right) E_{r} \otimes E_{s}$. В длинноволновом приближении этот член необходимо принимать во внимание. Именно он порождает связанные состояния в пустом волноводе [11].

ЗАМЕчАниЕ 3 . При определении $\chi_{0}$ мы никак не фиксировали функции $w_{1}^{\nu}, \ldots, w_{r}^{\nu}$. Мы считаем, что они образуют ортонормированный базис в собственном подпространстве задачи (3.3), отвечающем собственному значению (терму) $\varepsilon_{\perp}^{\nu}(x)$ с номером $\nu$, и гладко зависят от всех своих переменных. Такой базис, разумеется, не единственный, и его окончательный выбор удобно сделать при последуюшем построении асимптотических решений. Например, иногда в качестве $w_{j}^{\nu}$ можно взять собственные функции оператора момента $\hat{l}$, т.е. различать состояния внутри одного терма по проекциям орбитального момента в этих состояниях на ось трубки. Тогда матрица момента $\Lambda$ будет диагональной. Разумеется, можно менять базис и в пространстве спиноров; это бывает удобно сделать в случае, когда спин влияет на классическую динамику (в “средневолновом режиме"). Выбор нового базиса, очевидно, эквивалентен включению в формулу (4.1) после оператора $\hat{\chi}^{\nu}$ некоторой унитарной $(2 r \times 2 r)$-матрицы, зависяшей от $x$.

4.2. Эффективный гамильтониан продольного движения. "Быстрота" продольной моды, как отмечалось ранее, определяется параметром $h$. При $h \ll 1$ соответствуюшие состояния (в частности, уровни продольного движения) описываются квазиклассическими асимптотиками. Поскольку сильное электрическое или магнитное поля увеличивают продольную энергию частицы за время прохождения трубки, то, как мы уже отмечали в разделе 2 , параметр $h$ не может быть выбран произвольно и должен 
быть согласован с напряженностями внешних полей, входящих в задачу. Для математической строгости следует предполагать функциональную зависимость между параметрами $\mu, h$ и напряженностями полей. С физической точки зрения естественно говорить о величине различных слагаемых в (4.4) в зависимости от "быстроты" продольной моды и напряженности внешних полей. Для того чтобы решение с длиной волны $\sim h$ существовало, необходимо, чтобы работа эффективного поля $E_{\mathrm{eff}}(x)=-\partial v_{\mathrm{ext}} / \partial x-\partial \varepsilon_{\perp}(x) / \partial x-$ $\left\langle\partial_{x} \mathbf{R}, \partial \mathbf{A}_{0} / \partial t\right\rangle$ вдоль трубки не превосходила по порядку величины характерной кинетической энергии продольного движения $\varepsilon_{\|} \sim \mu^{2} / h^{2}$. Это влечет за собой следующие ограничения на внешние поля и “колебание" размеров сечения. Потенциал внешнего электрического поля будем считать равным нулю на одном из концов трубки, таким образом, $v_{\text {ext }}(\mathbf{R}(x), t)$ будет равен работе поля вдоль трубки. Отсюда следует, что внешний потенциал в безразмерных переменных имеет порядок $\mu^{2} / h^{2}$. Введем функцию $v_{\text {ext }}^{\prime}$, определенную равенством $v_{\text {ext }}=\left(\mu^{2} / h^{2}\right) v_{\text {ext }}^{1} ; v_{\text {ext }}^{1}$ принимает значения, не превосходящие единицы. В модельном примере (см. раздел 2) обсуждалась допустимая зависимость собственного значения $\varepsilon_{\perp}^{\nu}(x)$ задачи (3.3) от координаты $x$, согласованная с параметром $h$. Аналогично разделу 2 получаем $\varepsilon_{\perp}^{\nu}(x)=\varepsilon_{\perp}^{\nu}(0)+\left(\mu^{2} / h^{2}\right) \lambda^{\nu}(x)$. Ограничение на эффективное поле влечет за собой ограничение на характер зависимости магнитного поля от времени. А именно, мы будем полагать, что $\mathbf{H}=\mathbf{H}_{0}+(\mu / h) \mathbf{H}_{1}\left(t^{\prime}\right)$.

Следуя соображениям, изложенным в модельном примере (раздел 2), заменим “адиабатический" оператор импульса $\hat{p}=-i \mu \partial / \partial x$ на “динамический" $\hat{p}^{h}=-i h \partial / \partial x$, тогда $\hat{p}=(\mu / h) \hat{p}^{h}$, и затем “компенсируем" переопределение импульса, поделив адиабатический гамильтониан на $\mu^{2} / h^{2}$. Это приводит к переопределению классического импульса $p=(\mu / h) p^{h}$ и в силу (4.4) к следуюшей формуле для символа $\mathcal{L}^{\nu}\left(p^{h}, x, t, \mu, h\right)$ существенной части квантового әффективного гамильтониана $\hat{\mathcal{L}}^{\nu}$ продольного двиэсения:

$$
\begin{aligned}
\mathcal{L}^{\nu}= & \left.\frac{\left(p^{h}\right)^{2}}{2}+v_{\mathrm{ext}}^{1}(\mathbf{R}(x), t)+\phi(x, t)+\lambda^{\nu}(x)\right] E_{r} \otimes E_{s}+ \\
& +\frac{h^{2}}{\mu}\left[-E_{r} \otimes \frac{1}{2}\langle\boldsymbol{\sigma}, \mathbf{H}\rangle-\frac{1}{2}\left\langle\partial_{x} \mathbf{R}, \mathbf{H}\right\rangle \Lambda \otimes E_{s}+L_{s y}\right]+ \\
& +h\left[\left(\partial_{x} \Phi\right) \Lambda-\langle\mathbf{Y} \perp, \mathbf{H}\rangle\right] \otimes E_{s} p^{h}+ \\
& +\mu\left\langle\mathbf{Y}, \nabla v_{\mathrm{ext}}^{1}+\frac{\partial \mathbf{A}_{0}^{1}}{\partial t}+k\left(p^{h}\right)^{2} \mathbf{n}\right\rangle-\frac{h^{2} k(x)^{2}}{8} E_{r} \otimes E_{s},
\end{aligned}
$$

где $\mathbf{A}_{0}^{1}=(1 / 2)\left[\mathbf{R}, \mathbf{H}_{1}(t)\right], \phi(x, t)=(1 / 2) \int_{x^{*}}^{x}\left\langle\partial_{x} \mathbf{R}\left(x^{\prime}\right),\left[\mathbf{R}\left(x^{\prime}\right), \partial \mathbf{H}_{1}(t) / \partial t\right]\right\rangle d x^{\prime}$.

В уравнении (4.2) время $t$ оставлено размерным. Как и в модельном примере, характерное время прохождения трубки $T=(h / \mu) T_{0}$. Вводя безразмерное время $t^{\prime}$ согласно соотношению $t=T t^{\prime}=(h / \mu) T_{0} t^{\prime}$ и опуская штрихи, получаем искомое редуцированное уравнение на подзоне размерного квантования в следуюшем виде:

$$
i h \frac{\partial \psi^{\nu}}{\partial t}=\hat{\mathcal{L}}^{\nu} \psi^{\nu}, \quad \hat{\mathcal{L}}^{\nu}=\mathcal{L}^{\nu}\left(-i h \frac{\partial}{\partial x}, \stackrel{2}{x}, t, \mu, h\right)
$$


Вместе с формулами (4.1), (4.3) это уравнение при разных соотношениях между $\mu$ и $h$ определяет главный член асимптотических решений исходного уравнения (3.6), соответствующих всем состояниям, описанным в п. 2.9. При этом, как и в модельном примере, при соответствуюших соотношениях между $\mu$ и $h$ некоторые слагаемые в (4.5) могут быть опущены, а некоторые слагаемые из “адиабатической” поправки (иногда матричные) - переместиться в старшую часть эффективного “квазиклассического” гамильтониана, задаюшего, в частности, динамику классического движения частицы в тонкой трубке или, используя терминологию работы [14], нестандартные характеристики исходного уравнения (3.6). Описание такого “перемешения" и соответствуюших классических систем мы приводим в следуюшем, практически завершаюшем часть I этой работы пункте. Подробным конструкциям асимптотических решений уравнений (4.2) (и (3.6)) для перечисленных во введении задач и их приложениям будет посвящена часть II.

\section{3. Уравнения классической механики в нанотрубках с учетом спина и} кратности термов. Следуя соображениям, изложенным в разделе 2 , переразложим функцию (4.5). В случае $h \ll 1$ положим $\mathcal{L}^{\nu}=\mathcal{L}_{0}^{\nu}+h \mathcal{L}_{1}^{\nu}+\cdots$, где через $\mathcal{L}_{0}^{\nu}$ обозначены слагаемые, бо́льшие $h$, а через $h \mathcal{L}_{1}^{\nu}-$ слагаемые $\sim h$. Мы уже говорили в разделе 2 (см. также [13]) о том, что эти слагаемые позволяют полностью восстановить главный член квазиклассических асимптотик при $h \ll 1$, которые вне фокальных точек имеют вид ВКБ-решений (2.11). Их фазы определяются в результате интегрирования одномерной гамильтоновой системы $\dot{p}^{h}=\partial H_{\mathrm{eff}}^{h} / \partial x, \dot{x}=-\partial H_{\mathrm{eff}}^{h} / \partial p^{h}$ с классическим гамильтонианом $H_{\mathrm{eff}}^{h}\left(p^{h}, x\right)$, представляющим собой собственное значение матричного символа $\mathcal{L}_{0}^{\nu}$. При этом, если матрица $\mathcal{L}_{0}^{\nu}$ имеет различные собственные значения, то происходит квазиклассическое расщепление адиабатического терма, т.е. одному адиабатическому терму $H_{\text {eff }}^{\mu}$ могут соответствовать несколько различных классических гамильтонианов $H_{\mathrm{eff}}^{h}$. Векторная часть асимптотики $\psi^{\nu}$ находится из линейного уравнения "поляризации", в которое входит $\mathcal{L}_{1}^{\nu}$.

Рассмотрим следующие ситуации, соответствующие различным соотношениям между параметрами $\mu$ и $h$ (см. рис. 2 ).

1. Коротковолновый режим: $h=\mu$. Тогда

$$
\begin{aligned}
\mathcal{L}_{0}^{\nu}= & H_{\mathrm{eff}}^{h} E_{r} \otimes E_{s}, \quad H_{\mathrm{eff}}^{h}=\frac{\left(p^{h}\right)^{2}}{2}+v_{\mathrm{eff}}^{h}, \\
v_{\mathrm{eff}}^{h}= & v_{\mathrm{ext}}^{1}(\mathbf{R}(x), t)+\phi(x, t)+\lambda^{\nu}(x), \\
\mathcal{L}_{1}^{\nu}= & \left\langle\mathbf{Y}, \nabla v_{\mathrm{ext}}+\frac{\partial \mathbf{A}_{0}^{1}}{\partial t}+k\left(p^{h}\right)^{2} \mathbf{n}\right\rangle \otimes E_{s}+\left[\left(\partial_{x} \Phi\right) \Lambda-\langle\mathbf{Y} \perp, \mathbf{H}\rangle\right] \otimes E_{s} p^{h}+ \\
& +\left[-E_{r} \otimes \frac{1}{2}\langle\boldsymbol{\sigma}, \mathbf{H}\rangle-\frac{1}{2}\left\langle\partial_{x} \mathbf{R}, \mathbf{H}\right\rangle \Lambda \otimes E_{s}+L_{s y}\right] .
\end{aligned}
$$

Гамильтонова система в этом случае эквивалентна системе Ньютона $\ddot{x}=-\partial v_{\text {eff }}^{h} / \partial x$. Согласно оценкам п. 2.8, 2.10 главный член квазиклассических асимптотик, определяемый этими классическими уравнениями, должен давать хорошее приближение в задачах с внешними полями $|\mathbf{H}| \lesssim 1$ Тл, $E_{\text {ext }}=\left|\nabla v_{\text {ext }}\right| \lesssim 10^{-3} \mathrm{~B} /$ нм. 
2. Средневолновый режим: $h=\mu^{1 / 2}$. В этом случае

$$
\begin{aligned}
\mathcal{L}_{0}^{\nu} & =\left[\frac{\left(p^{h}\right)^{2}}{2}+v_{\mathrm{ext}}^{1}(\mathbf{R}(x), t)+\phi(x, t)+\lambda^{\nu}(x)\right] E_{r} \otimes E_{s}+W \\
W & =\left[-E_{r} \otimes \frac{1}{2}\langle\boldsymbol{\sigma}, \mathbf{H}\rangle-\frac{1}{2}\left\langle\partial_{x} \mathbf{R}, \mathbf{H}\right\rangle \Lambda \otimes E_{s}+L_{s y}\right] \\
\mathcal{L}_{1}^{\nu} & =\left(\left(\partial_{x} \Phi\right) \Lambda-\left\langle\mathbf{Y}{ }_{\perp}, \mathbf{H}\right\rangle\right) \otimes E_{s} p^{h} .
\end{aligned}
$$

Таким образом, здесь символ $\mathcal{L}_{0}^{\nu}$ - матричный, классические эффективные гамильтонианы - собственные значения матрицы $\mathcal{L}_{0}^{\nu}$. Очевидно, они представляются в виде суммы функции $\left(p^{h}\right)^{2} / 2+v_{\text {ext }}^{1}(\mathbf{R}(x), t)+\phi(x, t)+\lambda^{\nu}(x)$ и собственных значений матрицы $W$, вообше говоря, зависяших от $p^{h}$. Если они различны для всех $\left(p^{h}, x\right)$, то имеет место квазиклассическое расшепление адиабатического терма. Эти классические гамильтонианы, очевидно, оказываются зависяшими от спиновых слагаемых, поэтому спин частишы влияет на ее фазовую траекторию: энергия продольного движения в трубке, в отличие от полной и поперечной, сравнима со спиновой энергией.

Собственные значения матрицы $W$ зависят от вида поперечного сечения трубки и внешних полей. В качестве простейшего примера рассмотрим случай цилиндрически симметричного кристаллического потенциала $\left(y_{1}=\varrho \cos \phi, y_{2}=\varrho \sin \phi\right) v_{\text {int }}=v_{\text {int }}(\varrho)$, $w^{\nu}=e^{ \pm i \nu \phi} u^{\nu}(\varrho)$. Тогда $\mathbf{Y}=0$, собственные значения матрицы $\Lambda$ равны $\pm \nu, M^{1}=$ $M^{2}=0, \quad M^{0}$ - диагональная матрица с элементами $\pm \kappa \nu, \quad \kappa=2 \pi \int_{0}^{\infty}\left(\partial v_{\text {int }}(\varrho) / \partial \varrho\right) \times$ $\left(u^{\nu}(\varrho)\right)^{2} d \varrho$, и адиабатический терм расщепляется на четыре квазиклассических, задаваемых эффективными классическими гамильтонианами $\left(H_{\mathrm{eff}}^{h}\right)_{\uparrow \downarrow}^{ \pm}=\left(p^{h}\right)^{2} / 2+\left(v_{\mathrm{eff}}^{h}\right)_{\uparrow \downarrow}^{ \pm}$:

$$
\left(v_{\mathrm{eff}}^{h}\right)_{\uparrow \downarrow}^{ \pm}=v_{\mathrm{ext}}^{1}(\mathbf{R}(x), t)+\phi(x, t)+\lambda^{\nu}(x) \mp \frac{1}{2}\left\langle\partial_{x} \mathbf{R}, \mathbf{H}\right\rangle \nu+\sigma_{\uparrow \downarrow}\left|\frac{1}{2} \mathbf{H} \pm \nu \mu^{-1} \alpha \kappa \partial_{x} \mathbf{R}\right|,
$$

где $\sigma_{\uparrow \downarrow}= \pm 1$. При этом следует предполагать, что $\left|(1 / 2) \mathbf{H} \pm \mu^{-1} \alpha \kappa \nu \partial_{x} \mathbf{R}\right| \neq 0$, в противном случае имеет место эффект пересечения термов или смены кратности характеристик, где стандартное квазиклассическое приближение уже не работает (см., например, [23]). Гамильтонова система в этом примере также эквивалентна системе Ньютона с эффективным потенциалом $\left(v_{\text {eff }}^{h}\right)_{\uparrow \downarrow}^{ \pm}$. Квазиклассическое приближение должно работать для внешних полей $|\mathbf{H}| \lesssim 1$ Тл, $E_{\text {ext }}=\left|\nabla v_{\text {ext }}\right| \lesssim 10^{-5} \mathrm{~B} /$ нм. Отметим еще, что на уравнение (4.8) можно смотреть как на уравнение с операторнозначным символом (его операторный символ - матрица), и к нему можно повторно применить “операторную” редукцию. Этого вопроса мы еше коснемся в части II.

3. Длинноволновый режим: $h=1$. В отличие от рассмотренных ранее режимов, для сушествования “длинноволнового” приближения необходимо наложить дополнительные ограничения на величину магнитного поля. Формально это следует из наличия в гамильтониане (4.5) члена $\sim h^{2} / \mu$. Физическая причина заключается в следующем. Даже без учета спиновых эффектов продольная и поперечная моды связаны за счет взаимодействия магнитного поля с поперечным орбитальным моментом, которое описывается слагаемым $-(2 \mu)^{-1}\left\langle\partial_{x} \mathbf{R}, \mathbf{H}\right\rangle \Lambda \otimes E_{s}$. Поперечная энергия в данном случае значительно превосходит продольную, поэтому динамика продольного движения становится 
крайне чувствительной к малым изменениям поперечной энергии. Поперечная энергия, в свою очередь, меняется из-за изменения потока магнитного поля через сечение трубки при изменении угла наклона плоскости поперечного сечения трубки к вектору магнитного поля Н. При выбранных ранее магнитных полях $\sim 1$ Тл это взаимодействие приводит к разгону частицы до энергий, не согласованных с параметром $h$. Чтобы разгона частищы не было, нужно считать, что $\varepsilon_{\perp}^{\nu}(x)=\varepsilon_{\perp}^{\nu}(0)+\mu^{2} \lambda^{\nu}(x)$ и $\mathbf{H}_{0}=0$, $\mathbf{H}=\mu \mathbf{H}_{1}$. Это означает, что рассматриваются магнитные поля $\lesssim 10^{-2}$ Тл. Будем также предполагать, что безразмерная постоянная спин-орбитального взаимодействия $\alpha \sim$ $\mu^{2}$. Отсюда ясно, что в сушественную часть гамильтониана входят только слагаемые

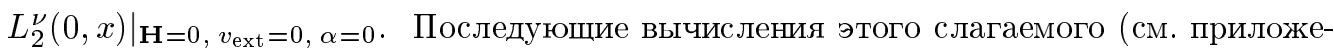
ние 3 ) приводят к последнему слагаемому в формуле (4.5). В результате мы получаем предельное редуцированное уравнение вида

$$
\begin{aligned}
& {\left[\left(-\frac{1}{2} \frac{\partial^{2}}{\partial x^{2}}+v_{\mathrm{ext}}^{1}(x)+\phi(x, t)\right) E_{r} \otimes E_{s}+\left(\partial_{x} \Phi\right) \Lambda \otimes E_{s}\left(-i \frac{\partial}{\partial x}\right)+W\right] \psi^{\nu}=0,} \\
& W=\left(\lambda^{\nu}(x)-\frac{k^{2}}{8}\right) E_{r} \otimes E_{s}-\frac{1}{2}\left\langle\partial_{x} \mathbf{R}, \mathbf{H}_{1}\right\rangle \Lambda \otimes E_{s}-E_{r} \otimes \frac{1}{2}\left\langle\boldsymbol{\sigma}, \mathbf{H}_{1}\right\rangle+\mu^{-1} L_{s y} .
\end{aligned}
$$

Квазиклассическое приближение здесь не применимо, и информацию о решениях редуцированного уравнения (точнее, системы уравнений) можно получать либо с помошью общих качественных методов, либо в результате численного интегрирования [7], [8], [26] . Как уже отмечалось, для оператора Гельмгольца в плоских одномодовых волноводах уравнение такого типа впервые было получено в работе [11], где была доказана, в частности, возможность организации в волноводе одного связанного состояния за счет подходящего выбора его кривизны. Уравнение, подобное (4.9), и некоторые следствия из него были получены в работах [7]. Мы также отмечали в разделе 2, что уравнение (4.9) работает в случае, когда поправка $L_{1}^{\nu}$ мала.

4. Ультракоротковолновый режим: $\mu^{3 / 2} \ll h \ll \mu$. В случае ультракороткого приближения допустимы более сильные внешние магнитное и электрическое поля, чем ранее, а именно $v_{\text {ext }} \sim \mu^{2} / h^{2},|\mathbf{H}(t)| \sim \mu / h,|\mathbf{H}| \lesssim 10$ Тл. Вводя обозначения $v_{\text {ext }}=$ $\left(\mu^{2} / h^{2}\right) v_{\mathrm{ext}}^{1}, \mathbf{H}(t)=(\mu / h) \mathbf{H}_{1}(t)$ и опуская штрихи, получаем

$$
\begin{aligned}
\mathcal{L}_{0}^{\nu}= & H_{\text {eff }}^{h} E_{r} \otimes E_{s}+\left(h^{2} \mu^{-2}\right) \varepsilon_{\perp}^{\nu}(x)+ \\
& +\mu\left(-\left\langle\mathbf{Y}_{\perp}, \mathbf{H}\right\rangle p^{h}+\left\langle\mathbf{Y}, \nabla v_{\text {ext }}+\frac{\partial \mathbf{A}_{0}}{\partial t}+k\left(p^{h}\right)^{2} \mathbf{n}\right\rangle\right), \\
\mathcal{L}_{1}^{\nu}= & \left(p^{h}\left(\partial_{x} \Phi\right)-\frac{1}{2}\left\langle\partial_{x} \mathbf{R}, \mathbf{H}_{1}\right\rangle\right) \Lambda- \\
& -\frac{1}{2}\left\langle\boldsymbol{\sigma}, \mathbf{H}_{1}\right\rangle+\mu^{-1} \alpha\left(M^{1} \otimes\left\langle\boldsymbol{\sigma}, \mathbf{n}_{1}\right\rangle p^{h}+M^{2} \otimes\left\langle\boldsymbol{\sigma}, \mathbf{n}_{2}\right\rangle p^{h}\right),
\end{aligned}
$$

где $H_{\mathrm{eff}}^{h}=\left(p^{h}\right)^{2} / 2+v_{\mathrm{ext}}(x)+\phi$. Несмотря на то что в этом случае символ $\mathcal{L}_{0}^{\nu}$ матричньй, его старшая часть есть $H_{\text {eff }}^{h} E_{r} \otimes E_{s}$. Тем не менее матричная добавка $\sim \mu>h$ может привести к расшеплению адиабатического терма и, в частности, к дополнительным слагаемым в фазе волновой функции $\sim \mu / h, \mu^{2} / h, \ldots$, вообще говоря, различным для каждого из состояний внутри адиабатического терма. Более детальное исследование расщепления в этом случае будет выполнено в части II работы. 
4.4. Моделирование эффективных потенциалов. Укажем в заключение на одно из элементарных, но любопытных свойств перечисленных выше уравнений, порожденных возможностями нанотехнологий: меняя геометрию трубки, помещенной в однородное әлектрическое поле, можнн моделировать различные әффективные одномерные потенииаль.

Рассмотрим сначала трубку постоянного сечения, ось которой - плоская кривая на плоскости $\left(r_{1}, r_{2}\right)$. Пусть электрическое поле с напряженностью $E_{\text {ext }}$ направлено вдоль оси $O x_{2}$. Тогда эффективньй потенциал имеет вид $\varphi(x)=v_{\text {ext }}(\mathbf{R}(x))=E_{\text {ext }} R_{2}(x)$. Если трубка изогнута не очень сильно по отношению к оси $r_{1}$, то $x \approx r_{1}$. Таким образом, выбирая ось трубки в виде подходящей кривой $r_{2}=r_{2}\left(r_{1}\right)$, можно моделировать потенциальную яму, двойную потенциальную яму и т.д.

В качестве примера неплоской трубки рассмотрим винтовую линию $\mathbf{R}(x)=$ $\left(\rho_{1} \cos \left(x / \sqrt{\rho_{1}^{2}+\rho_{2}^{2}}\right), \rho_{1} \sin \left(x / \sqrt{\rho_{1}^{2}+\rho_{2}^{2}}\right), \rho_{2} x / \sqrt{\rho_{1}^{2}+\rho_{2}^{2}}\right)\left(\rho_{1}=\right.$ const, $\rho_{2}=$ const - параметры) в поле $E_{\text {ext }}(0, \sin \alpha, \cos \alpha)$. Эффективный потенциал содержит осииллирующую и линейно возрастающую составляющие:

$$
\varphi(x)=-\sin \alpha E_{\mathrm{ext}} \rho_{1} \sin \left(x / \sqrt{\rho_{1}^{2}+\rho_{2}^{2}}\right)-x \cos \alpha E_{\mathrm{ext}} \rho_{2} / \sqrt{\rho_{1}^{2}+\rho_{2}^{2}}
$$

Если $\alpha=\pi / 2$, т.е. поле направлено перпендикулярно оси трубки, мы получаем периодический потенциал, а выведенные выше уравнения в первом приближении совпадают с уравнением Матье. В случае $\alpha=0$, т.е. когда поле направлено вдоль трубки, мы получаем уравнение Эйри. Более сложный пример - когда ось трубки служит обмоткой тора: в этом случае можно получать, в частности, почти периодические потенциалы. Подобных же результатов можно добиться, меняя вдоль оси толщину трубки.

ПРИЛОЖЕНИЕ 1

\section{Гамильтониан в криволинейных координатах}

Пусть $\mathbf{r}$ - радиус-вектор точки, гладкая вектор-функция $\mathbf{R}(x)$ определяет кривую $\gamma, x$ - натуральный параметр на $\gamma,\left\{\mathbf{n}_{1}, \mathbf{n}_{2}\right\}$ - некоторый ортонормированный базис в плоскости П $(x)$, пересекаюшей кривую $\gamma$ в точке $\mathbf{R}(x)$ ортогонально вектору $\partial_{x} \mathbf{R}$. Тогда в окрестности $\gamma$ имеем $\mathbf{r}=\mathbf{R}(x)+\mathbf{y}, \mathbf{y}=y_{1} \mathbf{n}_{1}(x)+y_{2} \mathbf{n}_{2}(x)$. Определитель матрицы Якоби, задающей переход от декартовых координат к координатам $\left(x, y_{1}, y_{2}\right)$, имеет вид $J=1-k\langle\mathbf{y}, \mathbf{n}\rangle$ (определение $\mathbf{n}, k$ и т.д. см. в п. 3.2), а определитель метрического тензора $G=J^{2}$. Введем "эффективное кручение" $\varkappa_{\mathrm{eff}}=-\left\langle\partial_{x} \mathbf{n}_{1}, \mathbf{n}_{2}\right\rangle$, тогда $\partial_{x} \mathbf{y}=-k\langle\mathbf{y}, \mathbf{n}\rangle \partial_{x} \mathbf{R}-\varkappa_{\mathrm{eff}} \mathbf{y}_{\perp}, \mathbf{y}_{\perp}=\left[\mathbf{y}, \partial_{x} \mathbf{R}\right]=y_{1} \mathbf{n}_{2}-y_{2} \mathbf{n}_{1},\left(\partial_{x} \mathbf{y}\right)^{2}=k^{2}\langle\mathbf{y}, \mathbf{n}\rangle^{2}+\varkappa_{\mathrm{eff}}^{2} \mathbf{y}^{2}$ Пользуясь этими соотношениями, легко вычислить компоненты метрического тензора:

$$
\begin{aligned}
& g_{11}=\left|\partial_{x} \mathbf{R}+\partial_{x} \mathbf{y}\right|^{2}=G+\varkappa_{\mathrm{eff}}^{2} y^{2} \\
& g_{12}=g_{21}=\left\langle\partial_{x} \mathbf{R}+\partial_{x} \mathbf{y}, \mathbf{n}_{1}\right\rangle=-\left\langle\partial_{x} \mathbf{n}_{1}, \mathbf{n}_{2}\right\rangle y_{2} \\
& g_{13}=g_{31}=\left\langle\partial_{x} \mathbf{R}+\partial_{x} \mathbf{y}, \mathbf{n}_{2}\right\rangle=\left\langle\partial_{x} \mathbf{n}_{1}, \mathbf{n}_{2}\right\rangle y_{1} \\
& g_{22}=g_{33}=1, \quad g_{23}=g_{32}=0
\end{aligned}
$$


Выберем базис $\left\{\mathbf{n}_{1}, \mathbf{n}_{2}\right\}$ способом, указанным в п. 3.2. Тогда $\varkappa_{\text {eff }}=0$. В уравнении (3.6) удобно перейти от функции $\Psi$ к функции $\Psi^{\prime}=J^{1 / 2} \Psi$. Такое переопределение волновой функции приводит к переопределению гамильтониана: $\mathcal{H}^{\prime}=J^{1 / 2} \mathcal{H} J^{-1 / 2}$.

В координатах $x, y_{1}, y_{2}$ имеем $J^{1 / 2} \Delta J^{-1 / 2}=J^{-1 / 2} \partial_{x} J^{-1} \partial_{x} J^{-1 / 2}+\Delta_{y}+k^{2} /(4 G)$. Отсюда после растяжения переменных $y=\mu y^{\prime}$ и отбрасывания штриха находим

$$
\begin{aligned}
& -\frac{\mu^{2}}{2} J^{1 / 2} \Delta J^{-1 / 2}= \\
& \quad=\left[-\frac{\mu^{2}}{2} \frac{\partial^{2}}{\partial x^{2}}-\frac{\Delta_{y}}{2}+\mu k\langle y, n\rangle \hat{p}^{2}-\frac{\mu^{2} k^{2}}{8 G}\right]+O\left(\mu^{2}\right)\left(-i \mu \frac{\partial}{\partial x}\right)+O\left(\mu^{3}\right) .
\end{aligned}
$$

Введем обозначения $\mathbf{A}_{0}=(1 / 2)[\mathbf{H}, \mathbf{R}(x)], \quad \mathbf{A}_{1}=(1 / 2)[\mathbf{H}, \mathbf{y}], \quad \mathbf{A}=(1 / 2)[\mathbf{H}, \mathbf{r}]=$ $\mathbf{A}_{0}+\mu \mathbf{A}_{1}$. Координатный базис $\left\{\partial_{x} \mathbf{r}, \mathbf{n}_{1}, \mathbf{n}_{2}\right\}$ отличается от ортонормированного базиса $\left\{\partial_{x} \mathbf{R}, \mathbf{n}_{1}, \mathbf{n}_{2}\right\}$ только переопределением первого базисного вектора $\partial_{x} \mathbf{r}=J \partial_{x} \mathbf{R}$. Принимая этот факт во внимание, для вектор-потенциала $\mathbf{A}$ получаем следующие тождества: $\langle A, \nabla\rangle=J^{-1}\left\langle\mathbf{A}, \partial_{x} \mathbf{R}\right\rangle \partial_{x}+\left\langle\mathbf{A}, \partial_{y}\right\rangle, \quad\left\langle\mathbf{A}_{1},-i \partial_{y}\right\rangle=(1 / 2)\left\langle\partial_{x} \mathbf{R}, \mathbf{H}\right\rangle \hat{l}$, $\left\langle\mathbf{A}_{1}, \partial_{x} \mathbf{R}\right\rangle=(1 / 2)\left\langle\mathbf{y}_{\perp}, \mathbf{H}\right\rangle, \quad\left\langle\mathbf{A}_{0}, \mathbf{A}_{1}\right\rangle=(1 / 2)\left\langle\left[\mathbf{A}_{0}, \mathbf{H}\right], \mathbf{y}\right\rangle$ (см. формулы (3.5)). Используя приведенные тождества и формулу (П.1), получаем первые три члена разложения (3.4) символа $\mathcal{H}$ по параметру $\mu \ll 1$.

ПРИЛОЖЕНИЕ 2

\section{Метод "операторного разделения" переменных}

Изложим схему нахождения коэффициентов разложения $L_{j}^{\nu}$ и $\chi_{j}^{\nu}$ символов операторов $\hat{L}^{\nu}$ и $\hat{\chi}^{\nu}$, введенных в (4.1), (4.2). В более обшей постановке речь идет о нахождении некоторых асимптотических по параметру $\mu \ll 1$ решений $\Psi=\left(\Psi^{1}, \ldots, \Psi^{s}\right)^{\mathrm{T}}(x, t), \mathbf{r}=$ $(x, y) \equiv\left(x_{1}, \ldots, x_{n}, y_{1}, \ldots, y_{m}\right) \in \mathbb{R}^{n+m}$, векторного уравнения типа уравнения Шредингера (3.6), порожденного матричным операторнозначным символом $\mathcal{H}=\| \mathcal{H}_{i j}(p, x$, $-i \partial / \partial y, y, \mu) \|, i, j=1, \ldots, s$.

Действие псевдодифференциального оператора задается с помошью " $\mu$-преобразования Фурье" [13], [21]:

$$
A\left(-i \mu \frac{\partial}{\partial x}, x\right) \varphi(x)=F_{p \rightarrow x}^{\mu}\left[A(x, p)\left[F_{x \rightarrow p}^{\mu} \varphi(x)\right](p)\right](x) ;
$$

прямое и обратное $\mu$-преобразования Фурье $F_{x \rightarrow p}^{\mu}$ и $F_{p \rightarrow x}^{\mu}$ определяются равенствами

$$
\begin{aligned}
& {\left[F_{x \rightarrow p}^{\mu} \varphi(x)\right](p)=\frac{1}{(2 \pi i \mu)^{n / 2}} \int_{\mathbb{R}_{x}^{n}} e^{-i\langle p, x\rangle / \mu} \varphi(x) d x,} \\
& {\left[F_{p \rightarrow x}^{\mu} \tilde{\varphi}(p)\right](x)=\frac{1}{(-2 \pi i \mu)^{n / 2}} \int_{\mathbb{R}_{p}^{n}} e^{i\langle p, x\rangle / \mu} \tilde{\varphi}(p) d p .}
\end{aligned}
$$

Важно подчеркнуть, что перед производными по "медленным" переменным $x$ стоит малый “адиабатический" параметр $\mu$, а перед производными по “быстрым" переменным $y$ мальй параметр отсутствует. Поэтому с точки зрения определения (П.2) символ $\mathcal{H}(p, x$, 
$-i \partial / \partial y, y, \mu)$ оператора $\widehat{\mathcal{H}}$ в свою очередь является оператором. Будем считать, что $\mathcal{H}(p, x,-i \partial / \partial y, y, \mu)$ разлагается в ряд по $\mu$ :

$$
\mathcal{H}(p, x,-i \partial / \partial y, y, t, h)=\mathcal{H}_{0}(p, x,-i \partial / \partial y, y, t)+\mu \mathcal{H}_{1}(p, x,-i \partial / \partial y, y, t)+\cdots .
$$

Мы предполагаем, что операторнозначный символ и его коэффишиенты разложения гладко зависящие от $p, x, t$ самосопряженные операторы, действуюшие в подходящем гильбертовом пространстве по переменным $y$ со скалярным произведением $\langle\cdot, \cdot\rangle_{y}$.

Ишем решение уравнения (3.6) в виде (см. (2.6))

$$
\Psi=\hat{\chi}^{\nu} \psi^{\nu} \equiv \chi^{\nu}(-i \mu \stackrel{1}{\partial} / \partial x, \stackrel{2}{x}, y, t, \mu) \psi^{\nu}(x, t, \mu),
$$

понимая в этом равенстве под $\psi^{\nu}=\left(\psi_{1}^{\nu}, \ldots, \psi_{k}^{\nu}\right)^{\mathrm{T}} k$-мерную волновую функцию некоторого выделенного терма (или выделенной “медленной” моды) с номером $\nu$ и кратностью вырождения $k$ и под символом "сплетающего" матричного псевдодифференциального оператора - гладко зависящую от всех своих переменных матричную функцию $\chi^{\nu}=\left\|\chi_{i j}^{\nu}(p, x, y, t, \mu)\right\|, \quad i=1, \ldots, s, \quad j=1, \ldots, k$, имеюшую разложение (2.7). При этом мы считаем, что вектор-функция $\psi^{\nu}$ удовлетворяет "эффективному редуцированному" уравнению (4.2), задаваемому матричным оператором $\hat{L}^{\nu}$ с гладким символом $L^{\nu}=\left\|L_{i j}^{\nu}(p, x, \mu)\right\|, i, j=1, \ldots, k$, и что символы $\chi^{\nu}$ и $L^{\nu}$ имеют асимптотические разложения (2.8) и (2.7), соответственно. Определив коэффициенты $\chi_{j}^{\nu}$ и $L_{j}^{\nu}$, мы сведем задачу построения асимптотических решений исходного уравнения к более простому (редуцированному) уравнению (4.2) для вектор-функции $\psi^{\nu}$, по которой исходная волновая функция $\Psi$ восстанавливается с помошью соотношения $\Psi=\hat{\chi}^{\nu} \psi^{\nu}$.

Подставляя функцию $\Psi=\hat{\chi}^{\nu} \psi^{\nu}$ в уравнение (3.6), получим $i T \mu \hat{\chi}^{\nu} \psi_{t}^{\nu}+i T \mu \hat{\chi}_{t}^{\nu} \psi^{\nu}=$ $\widehat{\mathcal{H}} \hat{\chi}^{\nu} \psi^{\nu}$. Используя (4.2), перепишем это уравнение в виде $\left(\hat{\chi}^{\nu} \hat{L}^{\nu}+i T \mu \hat{\chi}_{t}^{\nu}-\widehat{\mathcal{H}} \hat{\chi}^{\nu}\right) \psi^{\nu}=0$. Достаточным условием выполнения последнего равенства является операторное соотношение $\hat{\chi}^{\nu} \hat{L}^{\nu}+i T \mu \hat{\chi}_{t}^{\nu}-\widehat{\mathcal{H}} \hat{\chi}^{\nu}=0$. Перейдем в нем от операторов к символам, воспользовавшись при этом следуюшей формулой [21]: символ произведения операторов $A(-i \mu \stackrel{1}{\partial} / \partial x, \stackrel{2}{x}, \mu) B(-i \mu \partial / \partial x, \stackrel{2}{x}, \mu)$ равен $A(p-i \mu \partial / \partial x, \underset{2}{x}, \mu) B(p, x, \mu)$ (аккуратный вывод этой формулы для псевдодифференциальных операторов, описание условий для их символов и т.д. приведены в работах [21]). Это дает уравнение

$$
\begin{gathered}
\chi^{\nu}\left(p-i \mu \frac{\partial}{\partial x}, \stackrel{2}{x}, y, t, \mu\right) L^{\nu}(p, x, t, \mu)+i T \mu \chi_{t}^{\nu}(p, x, t, y, \mu)- \\
-\mathcal{H}\left(p-i \mu \frac{\partial}{\partial x}, \stackrel{2}{x}, y,-i \frac{\partial}{\partial y}, t, \mu\right) \chi^{\nu}(p, x, y, t, \mu)=0 .
\end{gathered}
$$

Его можно решать с помошью регулярной теории возмушений, разлагая входящие в него слагаемые в ряды по $\mu$. Собирая члены при $\mu^{0}=1$, получаем семейство зависяших от $x, p, t$ спектральных задач для самосопряженного оператора $\mathcal{H}_{0}(p, x,-i \partial / \partial y, y, t)$ :

$$
\mathcal{H}_{0}\left(p, x,-i \frac{\partial}{\partial y}, y, t\right) \chi_{0}^{\nu}(p, x, y, t)=\chi_{0}^{\nu}(p, x, y, t) L_{0}^{\nu}(p, x, t) .
$$


Будем считать, что асимптотика искомой функции $\Psi$ полностью определяется собственным значением (эффективным гамильтонианом или термом) $H_{\mathrm{eff}}^{\mu}(x, p, t)$, кратность которого равна $k$ и не зависит от $x, p, t$. Тогда

$$
L_{0}^{\nu}(p, x, t)=H_{\mathrm{eff}}^{\mu}(p, x, t) E,
$$

где $E$ - единичная матрица размерности $(k \times k)$, и матрица $\chi_{0}^{\nu}(p, x, y, t)$, составленная из ортонормированных вектор-столбцов - собственных функций оператора $\mathcal{H}_{0}$, отвечающих собственному значению $H_{\mathrm{eff}}^{\mu}(p, x, t),-$ это проектор на порожденное им собственное подпространство. Естественно считать, что $\chi_{0}^{\nu}(p, x, y, t)$ гладко зависит от всех своих аргументов. Кроме того, будем считать, что выполнено следующее условие: в некоторой фиксированной области $(p, x, t) \in \mathcal{M}$ значение $H_{\text {eff }}^{\mu}$ отделено равномерно по $(p, x, t)$ от других собственных значений или частей спектра $\mathcal{H}_{0}$ (если в спектре $\mathcal{H}_{0}$ имеется непрерывная компонента).

Собирая члены при $\mu$, получим неоднородные уравнения для $\chi_{j}^{\nu}$ и $L_{j}^{\nu}$ :

$$
\left(\mathcal{H}_{0}-H_{\mathrm{eff}}^{\mu} E\right) \chi_{j}^{\nu}=F_{j}-\mathcal{H}_{j} \chi_{0}^{\nu}+\chi_{0}^{\nu} L_{j}^{\nu}
$$

где $F_{j}$ зависят от $\chi_{0}^{\nu}, \ldots, \chi_{j-1}^{\nu}$ и $L_{0}^{\nu}, \ldots, L_{j-1}^{\nu} ;$ в частности,

$$
\begin{aligned}
F_{1}= & \widehat{\mathcal{D}} \chi_{0}^{\nu}, \\
F_{2}= & \widehat{\mathcal{D}} \chi_{1}^{\nu}-\mathcal{H}_{1} \chi_{1}^{\nu}+\chi_{1}^{\nu} L_{1}+i \sum_{j}\left[\frac{\partial \mathcal{H}_{1}}{\partial p_{j}} \frac{\partial \chi_{0}^{\nu}}{\partial x^{j}}-\frac{\partial \chi_{0}^{\nu}}{\partial p_{j}} \frac{\partial L_{1}}{\partial x^{j}}\right]+ \\
& +\frac{1}{2} \sum_{i, j}\left[\frac{\partial^{2} \mathcal{H}_{0}}{\partial p_{i} \partial p_{j}} \frac{\partial^{2} \chi_{0}^{\nu}}{\partial x^{i} \partial x^{j}}-\frac{\partial^{2} \chi_{0}^{\nu}}{\partial p_{i} \partial p_{j}} \frac{\partial^{2} \mathcal{H}_{\mathrm{eff}}^{\mu}}{\partial x^{i} \partial x^{j}}\right], \\
\widehat{\mathcal{D}}= & i T \frac{\partial}{\partial t}+i \sum_{j}\left[\frac{\partial \mathcal{H}_{0}}{\partial p_{j}} \frac{\partial}{\partial x^{j}}-\frac{\partial H_{\mathrm{eff}}^{\mu}}{\partial x^{j}} \frac{\partial}{\partial p_{j}}\right] .
\end{aligned}
$$

В силу самосопряженности оператора $\left(\mathcal{H}_{0}-H_{\text {eff }}^{\mu} E\right)$ и альтернативы Фредгольма условие разрешимости этого уравнения эквивалентно условиям ортогональности его правой части вектор-столбцам матрищы $\chi_{0}^{\nu}$. Отсюда получаем (ср. с [13]), $L_{j}^{\nu}=\left\langle\left(\chi_{0}^{\nu}\right)^{\mathrm{T}}, \mathcal{H}_{j} \chi_{0}^{\nu}-\right.$ $\left.F_{j}\right\rangle$. В частности,

$$
\begin{gathered}
L_{1}^{\nu}=\left\langle\left(\chi_{0}^{\nu}\right)^{\mathrm{T}}, \mathcal{H}_{1} \chi_{0}^{\nu}\right\rangle_{y}-i\left\langle\left(\chi_{0}^{\nu}\right)^{\mathrm{T}}, \frac{d \chi_{0}^{\nu}}{d t}\right\rangle_{y}-i\left\langle\left(\chi_{0}^{\nu}\right)^{\mathrm{T}}, \sum_{j}\left[\frac{\partial \mathcal{H}_{0}}{\partial p_{j}}-\frac{\partial H_{\mathrm{eff}}^{\mu}}{\partial p_{j}}\right] \frac{\partial \chi_{0}^{\nu}}{\partial x^{j}}\right\rangle_{y}, \\
\frac{d \chi_{0}^{\nu}}{d t}=T \frac{\partial \chi_{0}^{\nu}}{\partial t}-\sum_{j} \frac{\partial H_{\mathrm{eff}}^{\mu}}{\partial x^{j}} \frac{\partial \chi_{0}^{\nu}}{\partial p_{j}}+\sum_{j} \frac{\partial H_{\mathrm{eff}}^{\mu}}{\partial p_{j}} \frac{\partial \chi_{0}^{\nu}}{\partial x^{j}} .
\end{gathered}
$$

Считая, что $L_{1}^{\nu}$ имеет вид (П.7), можно найти поправку - матрицу $\chi_{1}^{\nu}=\left(\mathcal{H}_{0}-H_{\mathrm{eff}}^{\mu} E\right)^{-1} \times$ $\left(F_{1}-\mathcal{H}_{1} \chi_{0}^{\nu}+\chi_{0}^{\nu} L_{1}^{\nu}\right)$, фиксируя ее для определенности условием ортогональности вектор-столбцов матриц $\chi_{0}^{\nu}$ и $\chi_{1}^{\nu}$. Повторение этой процедуры приводит к вычислению $L_{m}^{\nu}$, $\chi_{m}^{\nu}$. Формулы (П.4), (П.7) и т.д. дают коэффициенты разложения символа редуцированного уравнения $(4.2)$ в любом порядке по $\mu$. 
ЗАмЕчАниЕ 4. Сформулированное выше условие эквивалентно отсутствию малых знаменателей в теории возмушений; его выполнение совершенно необходимо для асимптотических разложений. При выводе редуцированного уравнения (4.2) именно оно приводит к требованию учета всех собственных функций операторнозначного символа $\mathcal{H}_{0}$, отвечающих выделенному терму $H_{\mathrm{eff}}^{\mu}$. Как при проверке этого условия, так и при вычислении поправок $\chi_{j}^{\nu}$ сушественную роль играет ограниченность области $\mathcal{M}$ изменения переменных $p, x, t$. Если правые части $F_{j}$ растут, в частности, по $p$ при $p \rightarrow \infty$ (что имеет место в рассматриваемых в этой работе задачах), то малость $\mu$ в конкретных расчетах не гарантирует малости поправок $\chi_{j}^{\nu}$ и тем самым справедливости адиабатического приближения. Например, если $F_{1} \sim p^{2}$, то следует требовать $\mu p^{2} \ll 1$. Это неравенство ограничивает применение редуцированного уравнения (4.2) в “ультракоротком" случае.

ЗАмечАниЕ 5 . Слагаемое $-i\left\langle\left(\chi_{0}^{\nu}\right)^{\mathrm{T}}, d \chi_{0}^{\nu} / d t\right\rangle_{y}$ в символе $L_{1}^{\nu}$ может приводить к появлению дополнительной фазы у волновой функции - фазы Берри [27].

ПРИЛОЖЕНИЕ 3

\section{Вычисление коэффициентов разложения адиабатического гамильтониана}

Подставим оператор $\mathcal{H}_{0}$ из (3.4) в уравнение (П.3). Пусть $\left\{\eta_{1}, \eta_{2}\right\}$ - фиксированный ортонормированный базис в пространстве спиновых переменных, тогда под столбцами матрицы $\chi_{0}^{\nu}$ естественно понимать векторы (спиноры) вида $u_{m}^{\nu}(x, y) \eta_{i}, \quad m=1, \ldots, r$, $i=1,2$, и $\chi^{\nu}=\left\|u_{1}^{\nu}, \ldots, u_{r}^{\nu}\right\| \otimes E_{s}$. Функции $u_{m}^{\nu}(x, y) \eta_{i}$ образуют ортонормированный базис в собственном подпространстве, отвечающем $r$-кратно вырожденному собственному значению $\mathcal{H}_{\text {eff }}^{\mu}$ оператора

$$
\begin{gathered}
{\left[\frac{\mathcal{P}_{0}^{2}}{2}+v_{\text {ext }}(\mathbf{R}(x), t)+\sum_{j=1}^{2} \frac{\mathcal{P}_{j}^{2}}{2}+v_{\text {int }}(x, y)\right] u_{m}^{\nu}(x, y)=} \\
=\mathcal{H}_{\mathrm{eff}}^{\mu}(x, p, t) u_{m}^{\nu}(x, y),\left.\quad u_{m}^{\nu}(x, y)\right|_{\partial \Omega}=0
\end{gathered}
$$

причем условие Дирихле можно приближенно заменить на условие "мягких" стенок $u_{m}^{\nu}(x, y) \in L_{2}\left(\mathbb{R}_{y}^{2}\right)$. Последняя задача заменой $u_{m}^{\nu}=e^{i\left\langle\mathbf{y}, \mathbf{A}_{0}\right\rangle} w_{m}^{\nu}(x, y), \mathcal{H}_{\mathrm{eff}}^{\mu}(x, p, t)=$ $\mathcal{P}_{0}^{2} / 2+v_{\text {ext }}(\mathbf{R}(x), t)+\varepsilon_{\perp}^{\nu}(x)$ сводится к задаче $(3.3)$. Заметим, что $\chi_{0}^{\nu}$ оказывается не зависящей от $p$. Причина этого заключается в том, что в нулевом приближении в задаче (3.6) переменные разделяются.

Далее, имеем

$$
-i T\left\langle\chi_{0}^{\mathrm{T}}, \frac{d \chi_{0}}{d t}\right\rangle_{y}=\left[(W+Z) \mathcal{P}_{0}+i T\left\langle\mathbf{Y}, \frac{\partial \mathbf{A}_{0}}{\partial t}\right\rangle\right] \otimes E_{s},
$$

где $W_{m m^{\prime}}=-i\left\langle w_{m}^{\nu}, \partial_{x} w_{m^{\prime}}^{\nu}\right\rangle_{y}, \quad Z_{m m^{\prime}}=\left\langle w_{m}^{\nu},\left(\partial_{x}\left\langle\mathbf{y}, \mathbf{A}_{0}\right\rangle\right) w_{m^{\prime}}^{\nu}\right\rangle=-(1 / 2)\langle\mathbf{Y} \perp, \mathbf{H}\rangle-$ $k\langle\mathbf{Y}, \mathbf{n}\rangle\left\langle\partial_{x} \mathbf{R}, \mathbf{A}_{0}\right\rangle$. Пользуясь соотношениями

$$
\frac{\partial w^{\nu}}{\partial x}=\left(\left(\partial_{x} \Phi\right)\left[\mathbf{y}, \partial_{y}\right]-\frac{D^{\prime}}{D}\left\langle\mathbf{y}, \partial_{y}\right\rangle-\frac{D^{\prime}}{D}\right) w^{\nu}, \quad\left\langle w_{j}^{\nu},\left\langle\mathbf{y}, \partial_{y}\right\rangle w_{k}^{\nu}\right\rangle_{y}+\left\langle w_{j}^{\nu}, w_{k}^{\nu}\right\rangle_{y}=0
$$


получим, что $W=\mathcal{P}_{0}\left(\partial_{x} \Phi\right) \Lambda$. С помощью равенства (П.9) и соотношения $\left\langle\mathbf{Y},\left[\mathbf{A}_{0}, \mathbf{H}\right]\right\rangle=$ $\left\langle\mathbf{Y}_{\perp}, \mathbf{H}\right\rangle\left\langle\partial_{x} \mathbf{R}, \mathbf{A}_{0}\right\rangle-\left\langle\partial_{x} \mathbf{R}, \mathbf{H}\right\rangle\left\langle\mathbf{Y}_{\perp}, \mathbf{A}_{0}\right\rangle$ находим по формуле (П.7) символ $L_{1}^{\nu}$ в виде (4.4).

Из формул приложения 2 следует, что при определении $L_{2}^{\nu}$, вообще говоря, следует вычислить $\chi_{1}^{\nu}$. Покажем, что при сделанных в п. 4.3 предположениях относительно

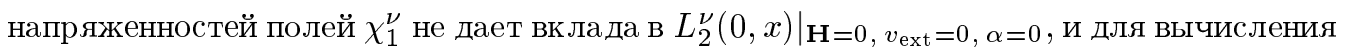
этого слагаемого нужно учитывать только "геометрическую" часть $\mathcal{H}_{2}$. Действительно, поскольку $\varepsilon_{\perp}^{\nu}(x)=\varepsilon_{\perp}^{\nu}(0)+\mu^{2} \lambda^{\nu}(x)$, то $\partial \chi_{0} / \partial x \sim \mu^{2}$, и при вычислении можно заменить $\partial \chi_{0} / \partial x$ нулем. Далее, имеем равенства $\partial \chi_{0}^{\nu} / \partial p=0,\left.\mathcal{H}_{1}(x, 0)\right|_{\mathbf{H}=0, v_{\text {ext }}=0, \alpha=0}=0$, $\left.L_{1}^{\nu}(x, 0)\right|_{\mathbf{H}=0, v_{\text {ext }}=0, \alpha=0}=0,\left.F_{1}(x, 0)\right|_{\mathbf{H}=0, v_{\text {ext }}=0, \alpha=0}=0,\left.\chi_{1}^{\nu}(x, 0)\right|_{\mathbf{H}=0, v_{\text {ext }}=0, \alpha=0}=0$, которые вместе с формулами (П.6) и приводят к требуемым выражениям для $\left.L_{2}^{\nu}(0, x)\right|_{\mathbf{H}=0, v_{\mathrm{ext}}=0, \alpha=0}$ и сушественной части гамильтониана продольного движения (4.5).

Благодарности. Мы благодарим за полезные дискуссии В. П. Маслова, К. В. Панкрашкина, И. В. Тютина и особенно В. А. Гейлера, прочитавшего рукопись и сделавшего ряд ценных замечаний. Работа выполнена при частичной поддержке грантов INTAS 00-257 и DFG 436 RUS 113/572/0-2.

\section{Список литературы}

[1] R. Saito, G. Dresselhaus, M. S. Dresselhaus. Physical Properties of Carbon Nanotubes. London: Imperial College Press, 1998; M. S. Dresselhaus, G. Dresselhaus, P. C. Eklund. Science of Fullerenes and Carbon Nanotubes. San Diego: Academic Press, 1996; И. В. Станкевич, М. В. Никеров, Д. А.Бочар. Усп. химии. 1984. Т. 53. № 7. С. 640.

[2] S. Iijima. Nature (London). 1991. V. 354. P. 56.

[3] V. Ya. Prinz, D. Grutzmacher, A. Beyer, C. David, B. Ketterer, E. Deckardt. Nanotechnology. 2001. V. 12. P. 1; L. A. Chernozatonskii, E. G. Gal'pern, N. R. Serebryanaya, I. V. Stankevich. Polymers of sungle-wall nanotubes: geometry, diffraction, patterns, and electronic spectrum modeling. In: AIP Proc. Eds. H. Kuzmany, J. Fink, M. Mehring, S. Roth. New York: AIP, 1999. Р. 284; Н. А. Поклонский, Е. Ф. Кисляков, Г. Г. Федорук, C. A. Вырко. ФТT. 2000. Т. 42. № 10. C. 1911.

[4] T. Hertel, G. Moos. Phys. Rev. Lett. 2000. V. 84. P. 5002.

[5] В. Ф. Гантмахер, И. Б. Левинсон. Рассеянные носители тока в металлах. М.: Наука, 1984.

[6] H. Jensen, H. Koppe. Ann. Phys. 1971. V. 269. P. 77; R. C. T. da Costa. Phys. Rev. A. 1981. V. 23. P. 1982; 1982. V. 25. P. 2893.

[7] Л. И. Магарилл, М. В. Энтин. ЖЭТФ. 2003. Т. 123. № 4. С. 867; M. V. Entin, L. I. Magarill. Phys. Rev. B. 2002. V. 66. P. 205308-1.

[8] K. Lin, R. L. Jaffe. Phys. Rev. B. 1996. V. 54. P. 5720; P. C. Schuster, R. L. Jaffe. Ann. Phys. 2003. V. 307. P. 132; P. Exner. J. Math. Phys. A. 1995. V. 28. P. 5323.

[9] B. De Witt. Phys. Rev. 1952. V. 85. P. 635.

[10] G. Panatti, H. Spohn, S. Teufel. Commun. Math. Phys. 2003. V. 242. № 3. P. 547; G. F. Dell'Antonio, L. Tenuta. J. Phys. A. 2004. V. 37. P. 5605; math-ph/0312034.

[11] В. П. Маслов. ДАН СССР. 1958. Т. 123. № 4. С. 631.

[12] Е. М. Воробьев, В. П. Маслов. ДАН СССР. 1968. Т. 179. С. 558; V. P. Maslov. Russ. Math. J. 2001. V. 8. № 1. P. 83.

[13] В. П. Маслов. Теория возмущений и асимптотические методы. М.: Изд-во МГУ, 1965.

[14] В. П. Маслов. УМН. 1983. Т. 38. № 6. С. 3. 
[15] M. Born, J. R. Oppenheimer. Ann. Phys. 1927. V. 84. P. 457; М. Борн, Х. Кунь. Динамическая теория кристаллических решеток. М.: ИЛ, 1958.

[16] Е. М. Лифиии, Л. П. Питаевский. Статистическая физика. Ч. 2. М.: Наука, 1978; В. С. Буслаев. ТМф. 1984. Т. 58. № 2. С. 233; УМН. 1987. Т. 42. № 6. С. 77; A. Fedotov, F. Klopp. Commun. Math. Phys. 2002. V. 227. № 1. P. 1; В. В. Белов. Изв. вузов. Математика. 1976. Т. 6. С. 13; J. Pedlosky. Geophysical Fluid Dynamics. New York: Springer, 1987.

[17] В. М. Бабич, В. С. Булдырев. Асимптотические методы в задачах дифракции коротких волн. М.: Наука, 1972.

[18] В. Г. Вакс. Межатомные взаимодействия и связь в твердых телах. М.: ИздАт, 2002.

[19] С. Ю. Доброхотов. ДАН СССР. 1983. Т. 269. № 1. С. 76; УМН. 1984. Т. 39. № 4. С. 125; Многофазовые асимптотические решения линейных и нелинейных уравнений в частных производных с малым параметром. Докт. диссертация. М.: Инст. пробл. мех. РАН, 1988; Л. В. Берлянд, С. Ю. Доброхотов. ДАН СССР. 1987. Т. 296. С. 80; S. Yu. Dobrokhotov, P. N. Zhevandrov. Russ. J. Math. Phys. 2003. V. 10. № 1. P. 1.

[20] В. В.Белов, С. Ю. Доброхотов, С. О. Синицын. Тр. Ин-та математики и механики УpO PAH. 2003. T. 9. № 1. C. S13; V. V. Belov, S. Yu. Dobrokhotov, S. O. Sinitsyn, T. Ya. Tudorovskiy. Докл. РАН. 2003. Т. 393. № 4. C. 460; V. V. Belov, S. Yu. Dobrokhotov, T. Ya. Tudorovskiy. Russ. J. Math. Phys. 2004. V. 11. № 1. P. 109.

[21] В. П. Маслов. Операторные методы. М.: Наука, 1973; М. В. Карасев, В. П. Маслов. УМН. 1984. Т. 39. № 6. С. 115; Нелинейные скобки Пуассона. Геометрия и квантование. М.: Наука, 1991.

[22] В. И. Арнольд. Математические методы классической механики. М.: Наука, 1974.

[23] Л. Д. Ландау, Е. М. Лифшич. Квантовая механика. Нерелятивистская теория. М.: Наука, 1989; В. В. Кучеренко. Изв. АН СССР. Сер. матем. 1974. Т. 38. № 3. С. 625; V. I. Il'ichov, V. A. Rivelis, V. S. Rabinovich, Y. V. Hoha. ДАН CССР. 1989. Т. 304. № 5. С. 1123; A. Gordon, J. E. Avron. Phys. Rev. Lett. 2000. V. 85. № 1. P. 34; J. E. Avron, A. Gordon. Phys. Rev. A. 2000. V. 62. P. 062504; Y. Colin de Verdier, B. Parisse. Commun. Math. Phys. 1999. V. 205. P. 459.

[24] П. К. Рашевский. Риманова геометрия и тензорный анализ. М.: Наука, 1967.

[25] T. Hertel, R. E. Walkup, P. Avouris. Phys. Rev. B. 1998. V. 58. P. 13870.

[26] В. А. Гейлер, И. Ю. Попов. ТМф. 1996. Т. 107. С. 427; В. А. Гейлер, В. А. Маргулис. Физ. техн. полупроводников. 1996. V. 9. Р. 1141; В. В. Грушин. Матем. заметки. 2004. T. 75. № 3. C. 360 .

[27] M. V. Berry. Proc. Roy. Soc. London. A. 1984. V. 392. P. 45.

Поступила в редакцию 22.IX.2003 г., после доработки 28.IV.2004 г. 